\title{
A novel grey-fuzzy-Markov and pattern recognition model for industrial accident forecasting
}

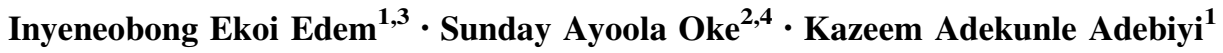

Received: 20 December 2015/ Accepted: 15 September 2017/Published online: 10 October 2017

(c) The Author(s) 2017. This article is an open access publication

\begin{abstract}
Industrial forecasting is a top-echelon research domain, which has over the past several years experienced highly provocative research discussions. The scope of this research domain continues to expand due to the continuous knowledge ignition motivated by scholars in the area. So, more intelligent and intellectual contributions on current research issues in the accident domain will potentially spark more lively academic, value-added discussions that will be of practical significance to members of the safety community. In this communication, a new grey-fuzzy-Markov time series model, developed from nondifferential grey interval analytical framework has been presented for the first time. This instrument forecasts future accident occurrences under timeinvariance assumption. The actual contribution made in the article is to recognise accident occurrence patterns and decompose them into grey state principal pattern components. The architectural framework of the developed grey-fuzzyMarkov pattern recognition (GFMAPR) model has four stages: fuzzification, smoothening, defuzzification and whitenisation. The results of application of the developed novel model signify that forecasting could be effectively carried out under uncertain conditions and hence, positions the
\end{abstract}

Sunday Ayoola Oke

sa_oke@yahoo.com

1 Department of Mechanical Engineering, Faculty of Engineering and Technology, Ladoke Akintola University of Technology, Ogbomoso, Oyo State, Nigeria

2 Department of Mechanical Engineering, Faculty of Engineering, University of Lagos, Lagos, Nigeria

3 Department of Industrial \& Production Engineering, Faculty of Technology, University of Ibadan, Ibadan, Nigeria

4 Industrial and Production Engineering Unit, Department of Mechanical Engineering, College of Engineering, Covenant University, Ota, Nigeria model as a distinctly superior tool for accident forecasting investigations. The novelty of the work lies in the capability of the model in making highly accurate predictions and forecasts based on the availability of small or incomplete accident data.

Keywords Forecasting - Manufacturing - Accidents · Fuzzy-grey-Markov $\cdot$ Pattern recognition

\section{Introduction}

An industrial accident refers to an undesirable, unanticipated and uncontrollable event potentially capable of producing injuries, losses of lives, asset destruction, and disturbance to social as well as economic activities or even leading to degradation of the environment in an industrial system. An accident is an occurrence triggered by human or non-human (i.e. entities, materials or emissions) in which the worker engaged in service to the industry may be injured. Every year, numerous literature reports are given, which declare an increasing number of industrial accidents globally. As a result of concerns to control accident occurrences, accident investigations are now a vital part of scientific reporting and a requirement by government agencies to all industrial organisations worldwide. Government policy stipulates proper reporting of accidents, its control and management. Hence globally, industrial managers are taking advantage of sound scientific studies to adopt models for their industries bearing in mind that an improperly planned accident control scheme could lead to substantial monetary losses due to accident claims.

For some years now, employing industrial forecasting models in accident forecasting relying on multiple factors has been justified by the fact that causal factors of accidents are attributed to human, equipment and managerial deficiencies 
(Cooke and Rohleder, 2006; Mohaghegh et al. 2009; Rathnayaka et al. 2011). Although proponents of models further justify the use of multiple causal factors, they also acknowledge that their degrees of interactions are also complex (Qureshi 2008; Stringfellow 2010). Unfortunately, since the institution of multivariate scales in the prediction and forecasting of industrial accidents, there has been a broad-spectrum of criticisms regarding the existence of unsatisfactory results. The problems of multivariate models are due to (1) inability to thoroughly capture the levels of interactions; (2) uncertainties; (3) randomness (Mao and Sun 2011); and (4) imprecision (Zheng and Liu 2009) inherent in accident causes and occurrences. In the sense of solving the problems attributed to multivariate models, the classical univariate prediction models (UPMs) were developed. UPMs are classical predictive models such as auto-regression and integrated moving average (ARIMA), exponential smoothing (ESM) and moving average (MA) adapted and applied in industrial accident forecasting (Kim et al. 2011; Kang et al. 2012; Aidoo and Eshun 2012). Scholars, however, also significantly criticised UPMs, in recent times. According to the literature, these mentioned models have not been entirely accurate in their applications to forecasting industrial accident occurrences. The drawbacks attributed to these models are as follows: take the case of MA, a constant mean of occurrence is assumed. However, this may not be true in practical instances of real-time occurrences. Another weakness of UPMs may be picked from the ARIMA model. It requires the availability of extensive data sizes to be able to make dependable predictions (Brockwell and Davis 2002). But this requirement of a large data size in the industrial world characterised by rapid information changes is a luxury that may be difficult to attain. In addition, accessing information in less industrially developed economies is quite challenging and such models may not be applicable in such environments.

Thus, for the aforementioned issues, we consider both UPMs and multivariate prediction models inappropriate for industrial accident forecasting. Yet there must be progress in the field. As the world experiences breakthrough in research on soft computing tool, more areas in science and technology are adopting these tools in their areas. Therefore, more recently, there has been a huge shift in focus towards accident occurrence prediction using non-traditional artificial intelligence (NTAI) forecasting approaches. With NTAI, new knowledge frontiers have been given birth to, expected to radically explode to benefit members of the industrial accident community. Models such as the artificial neural network (ANN) (Zheng and Liu 2009; Oraee et al. 2011), genetic algorithm (GA) (Farahat and Talaat 2012); grey (GM) (Jiang 2007; Lan and Ying 2014), grey-Markov model (Zhang 2010; Mao and Sun 2011; Huang et al. 2012a, b) and fuzzy time series models (FTSMS) (Khev and Yerpude 2015) have been employed in their original or modified forms for forecasting accidents which occur during mining, construction, transportation and processing activities. The results obtained from these model applications in industrial accident forecasting have also been very encouraging.

The organisation of the current work is as follows: the motivation and study objectives are stated in Sect. 1. A review of the literature is presented in Sect. 2. Sections 3, 4, 5, 6, and 7 are devoted to discussing the methodology of the proposed model. Model tests and validation results and discussion are given in Sect. 8. Conclusions concerning the model are shown in Sect. 9 alongside related future research directions.

\section{Related literature}

The application of grey, fuzzy and Markov principles in forecasting, as single concepts or merged together in different combination formats has begun to gain increasing popularity in recent years. In this section, a review of literature is given. Grey-fuzzy-Markov (GFM) forecasting technique is a hybrid model which combines the characteristics of the grey, fuzzy and Markov models. GFM models have been developed based on the understanding that hybrid models have greater forecasting potentials than single evaluation models ( $\mathrm{Li}$ and $\mathrm{Li}$ 2015). GFMs have found applications in areas such as electrical load analysis (Asrari et al. 2012) and biofuel production (Geng et al. 2015). The grey aspect of the GFM has its major focus on uncertainty inherent in sparsely available information (Deng 1982; Liu 2011). The model has been deeply explored for forecasting purposes and is evident by the development of several forms of it. Generally, a grey system can be mathematically expressed as

$a^{\prime \pm} \in[\widehat{a}, \grave{a}]$

$a^{\prime \pm}$ is a crisp value or an interval and exist as a component of a base set or interval $[\hat{a}, \vec{a}]$. Basic arithmetic, properties such as addition and multiplication as well as associative and commutative properties also apply in grey systems analysis (Hickey et al. 2001; Arroyo et al. 2011).

Two general forms of grey models, namely differential transfer function-based models (DTFM) and interval arithmetic-based models (IAM) have been mainly employed in areas such as energy consumption, finance and equipment degradation for crisp value forecasting (Kayacan et al. 2010; Tangkuman and Yang 2011; Mostafaei and Kardooni 2012) and interval forecasting (Garcia-Ascanio and Mate 2010; Zhao et al. 2014), respectively. DTFM involves the use of sequence operators (Liu et al. 2016) and unique mathematical representations to describe inputs and outputs under the assumption of exponential data 
behaviour. The most popularly employed grey model is the $\operatorname{GM}(1,1)$. It has been used in its pure form (Zhao et al. 2014; Tong 2016) or modified forms (Mao and Chirwa 2006; Jiang 2007; Zhang 2010; Mao and Sun 2011) for accident forecasting. The IAM involves the application of mathematical operations on grey intervals created from data to produce degeneration or interval forecast. The presence of the grey component, GFM, enables it to make accurate forecasts in the presence of limited and incomplete data, the fuzzy component of the model functions to eliminate the problem of vagueness and uncertainty in data (Chen and Hsu 2004; Kher and Yerpude 2015), while the Markov component deals with problems concerning fluctuating and random occurrences (Geng et al. 2015).

Generally, the procedure of GFM forecasting using the $\mathrm{GM}(1,1)$ as part of its component involves three major stages (Huang et al. 2012a, b; Li and Li 2015).

Stage 1: Building the grey model

This involves:

1. The creation of a time sequence for the set of available collection of industrial accident data

$x^{o}=\left(x_{1}^{o}, x_{2}^{o}, x_{3}^{o}, \ldots, x_{n}^{o}\right)$

2. Passage of the created sequence into an accumulated generating operation (AGO).

A modified sequence is obtained in the process

$x^{\prime}=\left(x_{1}^{\prime}, x_{2}^{\prime}, x_{3}^{\prime}, \ldots, x_{n}\right)$

$x_{i}^{\prime}=\sum_{k=1}^{i} x_{k}^{\prime}\{k=1,2,3, \ldots, n\}$

3. Establishment of a grey differential equation

$\frac{\mathrm{d} x_{i}^{\prime}}{\mathrm{d} t} a x_{i}^{\prime}(t)-b=0$

4. Solving to obtain the grey parameters $a$ and $b, \operatorname{GM}(1,1)$ forecast is then obtained as

$x_{k+1}^{G}=\left(x_{1}^{o}-(b / a) l^{a k}\right)$

Stage 2: Fuzzy classification of grey model errors.

This involves the linguistic classification of the percentage errors $e_{k}$ of each model forecast into $j$ number of classes carried out under the assumption of time invariance data behaviour (Sullivan and Woodward 1994). By the use of membership functions, the membership of $e_{k}$ in each fuzzy class $m\left[\mu\left(e_{k}, m\right), m: 1.2 .3, \ldots, j\right]$ is established. Huang et al. (2012a, b) and $\mathrm{Li}$ and $\mathrm{Li}$ (2015) employed the maximum membership principle $\max \left[\mu\left(e_{k}, m\right)\right]$ to establish the actual fuzzy class in which $e_{k}$ belongs.

Stage 3: Markov state transition

On the assumption that $m(m: 1,2,3, \ldots, j)$ exist as a Markov chain of states $s_{m}$ bounded by $\left(s_{m L}, s_{m U}\right)$, a Markov transition matrix which shows the probability of transition of the state in which $e_{k}$ belongs is $s\left(e_{k}\right)$, from its current state $y$ to another state $z\left(P_{y z}\right)$ in $t$ stepwise time order is set up:

$P^{t}\left[\begin{array}{cccc}P_{11}^{t} & P_{12}^{t} & P_{13}^{t} & P_{1 j}^{t} \\ P_{21}^{t} & P_{22}^{t} & P_{23}^{t} & P_{2 j}^{t} \\ P_{31}^{t} & P_{32}^{t} & P_{33}^{t} & P_{3 j}^{t} \\ \cdots & \cdots & \cdots & \cdots \\ P_{j 1}^{t} & P_{j 2}^{t} & P_{j 3}^{t} & P_{j j}^{t}\end{array}\right]$,

where

$P_{y z}^{t}=\frac{M_{y z}^{t}}{\sum_{z=1}^{j} M_{y z}^{t}}$

where $M_{y z}^{t}$ is the number of transitions from state $y$ to state $z$.

Stage 4: GFM model forecast

1. Based on the redistribution of fuzzy errors from the Markov transition technique, the fuzzified form of the forecast error $\mu\left(e_{n+1}, m\right)$ is then obtained as

$\mu\left(e_{n+1}, m\right)=P \mu\left(e_{k}, m\right)$

2. Subsequent defuzzification of $\mu\left(e_{n+1}, m\right)$ produces the crisp value of the forecast error $e_{n+1}$

$$
e_{n+t}=0.5 \sum_{m=1}^{j} \mu\left(e_{n+t}, m\right)\left(s_{m L}+s_{m U}\right)
$$

3. The GFM forecast for time step $t$ is finally obtained as

$$
Y_{n+t}=\frac{x_{n+t}^{G}}{\left(1-e_{n+t}\right)}
$$

Using this technique, Huang et al. (2012a, b) employed a dynamic grey model in detecting the dynamic trend of accident fatalities in the construction industry. $\mathrm{Li}$ and $\mathrm{Li}$ (2015) used an unbiased GM $(1,1)$ based GMF in also forecasting construction accidents.

This technique has been shown to improve on $\operatorname{GM}(1,1)$ and grey-Markov model prediction accuracies. However, the degree of prediction accuracies is limited. This is because the technique is actually directed at grey model prediction correction and as such, their prediction accuracies are directly dependent on the prediction accuracy of the grey model base. Thus, situations may exist in which GFM variants may not make be able to make forecasts that show significant improvement over those of the $\operatorname{GM}(1,1)$ base component.

In addition, Markov-chain transition analysis using the classical Markov state probability matrices and relations only provide general information on data dynamics. This is because the approach requires the availability of specific 
pre-existing states having similar characteristics to the current state occurrences. Thus, in using the technique, it may be difficult to detect sudden and previously non-existing changes in data behaviour. This is most obvious in situations of increased randomness and fluctuation in accident occurrences as well as limited availability of historical data. This renders the model incapable of providing satisfactory future transition probabilities in such situations.

This paper develops a grey-fuzzy-Markov industrial accidents forecast model for small or incomplete accident data availability situations using non-differential function grey interval analysis, fuzzy logic, variation conditioning and a state transition approach which aims to capture unique accident occurrence characteristics. The aim of the work is to create a standalone GFM model capable of making accurate industrial accidents forecasts.

The model's development is founded on its ability to recognise accident occurrence variation patterns. These patterns are then decomposed into certain principal pattern components identified in this paper. The results obtained from this knowledge is passed through a fuzzification process and rigorously treated to minimise noise in the fuzzy data. A decomposed state transition approach (DSTA) analogous to the classical Markov state transition approach is subsequently developed and used in detecting future accident vibrations and forecasts are then made in the process.

The validation of the model's existing value and future accident prediction capabilities is done using the in-fitsample and out-of-sample performance evaluation techniques, respectively. It is believed that this novel approach to industrial accident forecasting will aid proper anticipation, planning, control and management of future accident occurrences in industrial organisations on the one hand, and also provide a promising alternative tool to forecasting under uncertain conditions on the other.

The current paper makes a major contribution to the creation of a unique accident occurrence pattern recognition technique based on GFM inferences which acknowledge the significance of uncertainties. As such, the current paper contributes to the discussion on accident uncertainties, which has the interest of accident scholars and also grey-Markov-fuzzy theorists generally.

Industrial accidents forecasting, as argued in this paper, is central to the attainment of industry's stability and a guarantee to survive in the long run since litigation fees resulting from accidents could be reduced to the barest minimum through the adoption of a merit-driven forecasting technique. Nevertheless, the grey-fuzzy-Markov pattern recognition model has rarely been employed to improve forecasting and prediction of industrial accidents in industrial organisations. The authors found a number of papers applying only grey-fuzzy-Markov (Asrari et al. 2012; Geng et al. 2015) in the scientific literature with limited applications to the analysis of electrical and biofuel production, for instance. Industrial accident forecasting has not been tackled in grey-fuzzy-Markov literature. A key issue is that pattern recognition has been under-researched. This shows that the development of grey-fuzzy-Markov pattern recognition framework and the philosophical theory behind it in the context of industrial accidents is a sure gap filled in accident literature.

\section{Methodology}

The motivation for the creation of the grey-fuzzy-Markov pattern recognition prediction (GFMAPR) model arose from the observation on preliminary analysis that randomly summative and multiplicative relationships existed between industrial accidents data at different points within an existing data set. The need for the use of fuzzy logic was obvious as it was clearly difficult in employing classical mathematical approaches in understanding such data relationship.

\section{Acronyms, notations and model assumptions}

\section{Acronyms}

\section{CPI Comparative performance index}

CPS Cumulative pattern swing

CVSM Cumulative variation swing magnitude

DSTA Decomposed state transition approach

FAC Forecast acceptability criterion

GFMAPR Grey-fuzzy-Markov pattern recognition

MDR Multiplicative data relationship

SDR Summative data relationship

PE Performance evaluation

VPCPS Variation principal component pattern swing

FGM Fuzzy-grey-Markov

Notations

$x \quad$ Available historical data

$d \quad$ First-level historical data variation

$z \quad$ Cumulative sum of $d$

$s \quad$ Markov states for periodic $z$ values

$s^{L} \quad$ Lower Markov state bound

$s^{u} \quad$ Upper Markov state bound

$\sigma \quad$ Markov states partitioning index

$\omega \quad$ First-level variation Markov state width

$\mu \quad$ Fuzzy membership value for Markov states

$\hat{x} \quad$ SDR analysis forecast 
$\delta \quad$ Periodic change in historical data variation

$\bar{D} \quad$ Second-level variation class

$r_{j}^{L} \quad$ Future second-level variation value for $r$ pattern swing

$\rho(q) \quad$ Polarity of variable $q$

$\rho^{i}(q) \quad$ Variable $q$ of positive or negative polarity $i$

$k_{\max } \quad$ Maximum number of Markov states

$E_{\text {cur }}^{C} \quad$ Current escalation cumulative swing magnitude

$U_{\text {cur }}^{C} \quad$ Current closure-lag cumulative swing magnitude

$\Gamma \quad$ Proximity score index

$\lambda$ Cumulative variation pattern swing magnitude

$:=\quad$ Equal by definition

$\leftrightarrow \quad$ Same as

$\uparrow \quad$ Not the same as

\# Cardinality of set

$i, j, k, m$ Various subscripts representing the periodic state, condition or value of any described variable

○

Superscript which denotes variables of MDR analysis

Model assumptions

1. Available historical data are randomly occurring and of non-stagnant pattern occurrence feature.

2. Information available for analysis is unique to that system and different in characteristics and behaviour to that of other systems.

3. There is always a summative or multiplicative variation relationship or both existing within any available historical dataset.

4. Second-level variations process has strictly non-static characteristics.

5. A time invariance nature of data exists (Sullivan and Woodward 1994).

\section{GFMAPR: the concept}

To be able to develop GFMAPR, two grey-fuzzy-Markov analysis methods, namely summative data relationship (SDR) analysis and multiplicative data relationship (MDR) analysis were carried out on two differently prepared versions of historical data. Grey probable forecasts were subsequently generated from the SDR forecast interval and cross-checked with MDR forecast interval expectations. Based on a set criterion of acceptability, probable forecasts which fell within SDR and MDR interval intersection space were further analysed using a whitenisation procedure to produce the crisp forecast. An outline of the GFMAPR concept is presented in Fig. 1. The procedure for determining the SDR and MDR will be discussed independently in subsequent sections of this paper.

\section{Procedure for the SDR determination}

The analysis to determine the SDR is discussed in this section using the outline in Fig. 1.

\section{SDR preparation}

Data preparation is the first stage in the SDR process. This stage involves the application of the AGO. At this stage, available historical data $x_{(1, n)}\left\{x_{i}: i=1,2,3, \ldots, n\right\}$ were converted into a set of values $z_{i}\{i=1,2,3, \ldots, n\}$ by a cumulative summation of their variations. This is the firstlevel variation analysis.

$z_{i}= \begin{cases}z_{i-1}+d & \{i=2,3, \ldots, n\} \\ d_{i} & \{\text { otherwise }\}\end{cases}$
$d_{i}= \begin{cases}x_{i}-x_{i-1} & \{i=2,3, \ldots, n\} \\ 0 & \text { \{therwise }\}\end{cases}$

\section{Creation of summative variation states}

Based on results obtained, a set of grey states $s_{k}$ was created to accommodate $z_{i}$. In creating $s_{k}$, consideration was given to the dynamic nature of $x_{i}$ evidenced in $z_{i}$ To be able to reflect the current characteristics of data, a position influenced state interval $\omega$, assumed uniform for all states was created

$\omega=\left(\sum_{i=1}^{n-1} p^{2}\left|z_{i}-z_{i+1}\right|\right) / \sigma \sum_{i-1}^{n-1} p^{2}$

where $\omega$ is the parameter which is used to adequately express the relationship between $x_{i}$ and $x_{i+1}$. Thus, the accuracy of GFMAPR is strongly dependent on $\omega$. Obtaining this value requires that two values $p$ and $\sigma$ must be supplied in Eq. (14). $p$ indicates the position characteristic of $x_{i}$ in the data set and $\sigma$ is the set partitioning index. $p$ and $\sigma$ have to be determined.

Following preliminary investigation of some industrial accident occurrence data, $p$ was taken in this work as a constant and fixed as

$p=i^{2}$

$\sigma$ was considered a variable and arbitrarily fixed at an initial value of $4 . s_{k}$ were thus created as follows:

$s_{k}:=\left(s_{k}^{L}, s_{k}^{U}\right)$

$s_{k}:=\left\{\begin{array}{cc}\left(z_{1}, z_{1}+\omega\right) & \{k=1\} \\ \left(s_{k-1}, s_{k-1}+\omega\right) & \{\text { otherwise }\}\end{array}\right.$

The state creation procedure is terminated at $k_{\max }$ given that $s_{k}^{U} \geq \max \left(z_{i}\right)$. To reduce the problem of overestimating the terminal state, $s *\left[s *=s_{k}\left(k=k_{\max }\right)\right]$ 
Fig. 1 Outline of the FGMaPR forecast concept

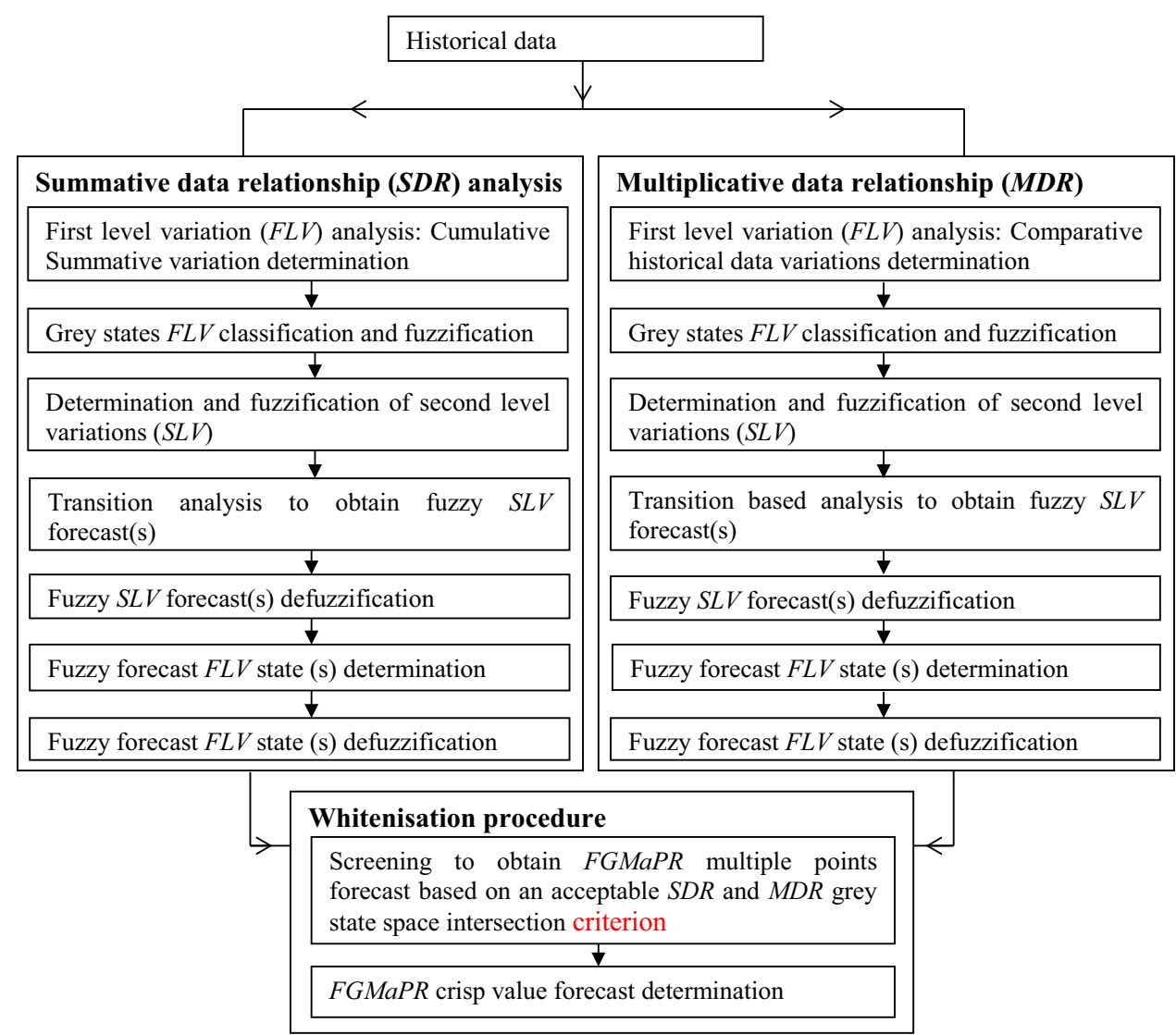

is defined as

$s *:=\left(s_{k}^{L}, \max \left(z_{i}\right)\right)\left\{k=k_{\max },\left(\left(\max \left(z_{i}\right)-s_{k}^{L}\right) / \omega\right)>0.75\right\}$

\section{Fuzzification and reclassification of summative variations}

$\left[s_{1}^{L}, s_{k}^{U}\left(k=k_{\max }\right)\right]$ was adopted as the initial universe of discourse in this work. At this stage $\sigma=4$ is not considered as the value that provides the most satisfactory interval

The need to locate $z_{i}$ more precisely within $s_{k}$ necessitated the fuzzification of $z_{i}$. Due to its simplicity and ease of use, the triangular fuzzy membership function was adopted for the fuzzification procedure in this work. Membership functions for derived $s_{k}$ membership classes are presented in the following equations:

$$
\begin{aligned}
& \mu\left(z_{i}, k\right)=\left\{\begin{array}{l}
1\left\{z_{i}<a_{k}, k=1\right\} \\
\left(z_{i}+0.25 a_{k}-1.25 a_{k+1}\right)\left(1.25\left[a_{k}-a_{k+1}\right]\right)^{-1}\left\{a_{k} \leq z_{i} \leq a_{k+1}, 1 \leq k \leq k_{\max }-1\right\} \\
0 \quad \text { otherwise }\}
\end{array}\right. \\
& \mu\left(z_{i}, k+1\right)=\left\{\begin{array}{l}
0 \quad\{\text { otherwise }\} \\
\left(a_{k}-z_{i}\right)\left(1.25\left[a_{k}-a_{k+1}\right]\right)^{-1}\left\{a_{k} \leq z_{i} \leq a_{k+1}, 1 \leq k \leq k_{\max }-1\right\} \\
1\left\{z_{i}>a_{k}, k=k_{\max }\right\}
\end{array}\right.
\end{aligned}
$$

width $\omega$. The procedure that modifies the universe of discourse by searching for the most satisfactory $\omega$ was undertaken. This is discussed later. where $\mu\left(z_{i}, k\right)$ represents the fuzzy membership values of $z_{i}$ in $s_{k}$ and $a_{k}$ are the midpoints of fuzzy sets $s_{k}\left(i=1,2,3, \ldots, n ; k=1,2,3, \ldots, k_{\max }\right)$. 
The actual state in which $z_{k}$ belongs after fuzzification was subsequently relocated as

$s_{i}^{\prime}=s_{k}\left[\max \left(\mu\left(z_{i}, k\right)\right)\right]$

In-fit-sample forecasts produced by this state classification procedure was then obtained using the following equation:

$\hat{x}_{i}= \begin{cases}\bar{s}_{i}+x_{i} & \{i=1\} \\ \bar{s}_{i}^{\prime}-\bar{s}_{i-1}^{\prime}+\hat{x}_{i-1} & \text { \{otherwise }\}\end{cases}$

where $\overline{s_{i}}$ is the midpoint value of $s_{i}^{\prime}$.

Equation (22) expresses the summative relationship which exists within any given set of historical data related to their variations.

Although the approach establishes that a relationship for a historical data set existing within period $n$, it cannot be directly employed in forecasting for periods existing outside the historical data window due to the time-invariant data assumption made. The rest of the work is directed at the analysis of the time-invariant model towards obtaining GFMAPR future value forecasts.

\section{Second-level variation analysis and classification of degree of variation}

Let variation of $z_{i}$ from its current state $s_{i}^{\prime}$ of state number $k\left(s_{k}=s_{i}^{\prime}\right)$ to its terminal state $s_{i+1}^{\prime}$ of number $k\left(s_{k}=s_{i+1}^{\prime}\right)$ be represented by $\delta_{i, i+1}$. That is,

$\delta_{i, i+1}=k\left(s_{k}=s_{i}^{\prime}\right)-k\left(s_{k}=s_{i+1}^{\prime}\right) \quad\{i=1,2,3, \ldots, n-1\}$

$\delta_{i, i+1}$ indicates the periodic change in first-level variation within data. Subsequently, the relationship that exists within $\delta_{i, i+1}$ was investigated.

\section{Fuzzy classification of the second-level variations}

Preliminary investigation led the classification of the second-level variation (change in the first level) variation $\delta_{i, i+1}$ into four fuzzy classes $C_{b}(b=1,2,3,4)$ namely: small-level variation (SV), small to medium level variation (SMV), medium to large level variation (MLV) and large level variation $(\mathrm{LV})$. Fuzzy classes based on a trapezoidal membership function $\delta_{i, i+1} Z$ were created for these linguistic classes.

$$
\begin{aligned}
& \mu\left(\left|\delta_{i, i+1}\right|, C_{1}\right) \\
& = \begin{cases}1 & \left\{\left|\delta_{i, i+1}\right| \leq h_{1} m\right\} \\
-10\left(\left[\frac{\delta_{i, i+1} \mid}{m}\right]-h_{1}\right)+1 & \left\{h_{1} m<\left|\delta_{i, i+1}\right| \leq\left(h_{1}+0.1\right) m\right\} \\
0 & \{\text { otherwise }\}\end{cases}
\end{aligned}
$$

$$
\begin{aligned}
& \mu\left(\left|\delta_{i, i+1}\right|, C_{b}[b=2,3]\right) \\
& = \begin{cases}10\left(\left[\left|\delta_{i, i+1}\right| / m\right]-h_{b}-1\right) & \left\{h_{b-1},\left|\delta_{i, i+1}\right| \leq\left(h_{b-1}+0.1\right) m\right\} \\
1 & \left\{\left(h_{b-1}+0.1\right) m<\left|\delta_{i, i+1}\right| \leq h_{b} m\right\} \\
-10\left(\left[\left|\delta_{i, i+1}\right| / m\right]-h_{b}\right)+1 & \left\{h_{b} m<\left|\delta_{i, i+1}\right| \leq\left(h_{b}+0.1\right) m\right\} \\
0 & \text { otherwise }\}\end{cases}
\end{aligned}
$$

$\mu\left(\left|\delta_{i, i+1}\right|, C_{4}\right)$

$= \begin{cases}10\left(\left[\left|\delta_{i, i+1}\right| / m\right]-h_{3}\right) & \left\{h_{3} m<\left|\delta_{i, i+1}\right| \leq\left(h_{3}+0.1\right) m\right. \\ 1 & \left.\left\{\left|\delta_{i, i+1}\right|>h_{3}+0.1\right)\right\} \\ 0 & \text { otherwise }\}\end{cases}$

$h_{b}=0.25 b$

$m=\max \left|\delta_{i, i+1}\right|-\min \left|\delta_{i, i+1}\right| \quad\left\{\delta_{i, i+1} \neq 0\right\}$

In addition, employing the maximum membership principle, the actual fuzzy class in which $\left|\delta_{i, i+1}\right|$ belongs was obtained as

$C\left(\left|\delta_{i, i+1}\right|\right)=\max \left(\mu\left(\left|\delta_{i, i+1}\right|, C_{b}\right)\right)$

Employing Eq. (29), crisp representatives, $\bar{D}\left(\delta_{i, i+1}\right)$ of $C\left(\left|\delta_{i, i+1}\right|\right)$ were subsequently employed for further analysis.

$\bar{D}=\left\{\begin{array}{cl}b & \left\{\left(\left|\delta_{i, i+1}\right|\right)=C_{b}^{*} ; \delta_{i, i+1}>0\right\} \\ -b & \left\{\left(\left|\delta_{i, i+1}\right|\right)=C_{b}^{*} ; \delta_{i, i+1}<0\right\} \\ 0 & \text { atherwise }\}\end{array}\right.$

$C_{1}^{*}= \begin{cases}\left\{C_{1}\right\} & \left\{\# C_{1}>0\right\} \\ \left\{C_{2}\right\} & \text { otherwise }\}\end{cases}$

$C_{t}^{*}= \begin{cases}C_{t} & \left\{\#\left\{C_{t}, t=2,3\right\}>0\right\} \\ C_{t-1} \cup C_{t+1} & \text { \{otherwise } t=2,3\}\end{cases}$

$C_{4}^{*}= \begin{cases}\left\{C_{4}\right\} & \left\{\# C_{4}>0\right\} \\ \left\{C_{3}\right\} & \text { otherwise }\}\end{cases}$

Expressions (31), (32) and (33) were developed to account for situations of shock occurrences. In such cases, it is possible for intermediate variation classes to be nonexistent, in the presence of higher variation classes. The relations function in smoothening variation levels.

It can be observed that although the $S L V$ was fuzzified, crisp values were employed as the representation of each linguistic class or interval. This was made necessary due to the need to undertake grey analysis on the set of fuzzy classes.

\section{Treatment of data to account for static second-level non-variation situations}

The non-static $S L V$ assumption employed in this study allows consideration to be given only to non-zero $\delta_{i, i+1}$ as can be observed in expressions (28). However, situations in 
which $\delta_{i, i+1}=0$ do occur and non-zero $\bar{D}\left(\delta_{i, i+1}\right)$ equivalents must exist for such situations. To surmount this challenge, smoothing procedures for three different second-level non-variation scenarios were introduced. These are presented in "Appendix A".

At the end of the smoothing procedure, let the smoothened values of $\bar{D}_{j}$ be represented by $\bar{D}_{j}^{\prime \prime}$.

\section{Pattern principal component analysis}

This stage of the SDR analysis was undertaken in five substages, namely (i) identification of variation principal component pattern swing (VPCPSs) from the grey form of the fuzzy data obtained from previous analysis. (ii) Determination of directions for expected future VPCSPS swings. (iii) VPCSPS swing value adjustments. (iv) Determination of expected future VPCSP values. (v) Verification and adjustment of expected future VPCSPs values. These are treated in this section.

Note that the term "pattern swing" will be used interchangeably with VPCSPs in the course of this discussion.

\section{Identification of data variation principal component pattern swings}

Based on the preliminary analysis of several industrial accidents historical data and information, the observation that industrial accidents mostly exhibit randomly trending or fluctuating characteristics or a combination of both were made. Another major feature also observed was that of the presence of various degree randomly occurring shocks within data. Figure 2 shows a cross section of real-time industrial accident occurrences.

Employing these observations, five unique fuzzy VPCPSs which exist in any characteristic variation curve were identified as open $\left(O^{L}\right)$, escalation $\left(E^{L}\right)$, exact-closure $\left(C^{L}\right)$, closure-lag $\left[U^{L}\right]$ and closure-lead $\left(V^{L}\right)$. Letting each $\bar{D}_{j}^{\prime \prime}$ be the periodic swing magnitude for all periods $j$ the various VPCPSs are defined below:

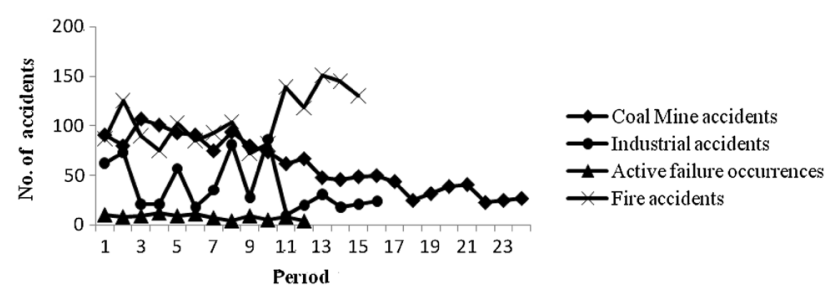

Fig. 2 Cross section of real-time industrial accidents occurrence patterns Sources: (Kher and Yerpude 2015; Okoh and Haugen 2014; Shin 2013; Docstoc 2013)
1. Open pattern swing: This is taken in this work as the next variation swing in the time $j$ given that the previous cumulative variation swing magnitude $(\mathrm{CVSM}) \lambda_{j-1}$ is equal to zero. $O^{L}$ can exist at variation curve points occurring immediately after two swings of equal magnitude and opposite poles have offset each other. It can also occur as the sum of swing magnitudes $\bar{D}_{j}^{\prime \prime}$ and $\lambda_{j-1}$ given that both have opposing poles and the latter is of lesser absolute magnitude.

$$
O_{j}^{L}= \begin{cases}\bar{D}_{j}^{\prime \prime} & \left\{\lambda_{j-1}=0\right\} \\ V_{j}^{L} & \left\{\left|\lambda_{j-1}\right| \leq\left|\bar{D}_{j}^{\prime \prime}\right|, \lambda_{j-1} \neq 0, A\right\} \\ 0 & \text { otherwise }\}\end{cases}
$$

2. Escalation swing: this is a type of variation swing $\bar{D}_{j}^{\prime \prime}$ that occurs when $\lambda_{j-1} \neq 0$ with both having the same polarity.

$E_{J}^{L}= \begin{cases}\frac{\bar{D}_{j}^{\prime \prime}}{0} & \frac{\left\{\lambda_{j-1} \neq 0, B\right\}}{\{\text { otherwise }\}}\end{cases}$

3. Exact-closure swing: this is expressed as the value of $\bar{D}_{j}^{\prime \prime}$ with magnitude equal to $\lambda_{j-1}$ but opposite in polarity.

$$
C_{j}^{L}= \begin{cases}-\lambda_{j-1} & \left\{\left|\lambda_{j-1}\right| \leq\left|\bar{D}_{j}^{\prime \prime}\right|, \lambda_{j-1} \neq 0, A\right. \\ 0 & \{\text { otherwise }\}\end{cases}
$$

4. Closure-lag swing: this is the value of $\bar{D}_{j}^{\prime \prime}$ with magnitude less than $\lambda_{j-1}$ but opposite in polarity.

$$
U_{j}^{L}= \begin{cases}\bar{D}_{j}^{\prime \prime} & \left\{\left|\lambda_{j-1}\right|>\left|\bar{D}_{j}^{\prime \prime}\right|, \lambda_{j-1} \neq 0, A\right. \\ 0 & \{\text { otherwise }\}\end{cases}
$$

5. Closure-lead swing: this is the value of $\bar{D}_{j}^{\prime \prime}$ with magnitude greater than $\lambda_{j-1}$ but opposite in polarity

$$
V_{j}^{L}= \begin{cases}\left(\lambda_{j-1}+\bar{D}_{j}^{\prime \prime}\right) & \left\{\left|\lambda_{j-1}\right|<\left|\bar{D}_{j}^{\prime \prime}\right|, \lambda_{j-1} \neq 0, A .\right. \\ & \text { \{otherwise }\}\end{cases}
$$

Simultaneously, pattern swing occurrence indicators $F\left\{r_{j}^{L}\right\}$ were obtained as

$$
\left\{F\left\{r_{j}^{L}\right\}=1 \quad \begin{array}{rl}
\left\{r_{j}^{L} \neq 0\right\} \\
0 & \{\text { otherwise }\}
\end{array}\right.
$$

where, $A=\rho\left(\bar{D}_{j}^{\prime \prime}\right) \uparrow \rho\left(\lambda_{j-1}\right)$

$B=\rho\left(\bar{D}_{j}^{\prime \prime}\right) \leftrightarrow \rho\left(\lambda_{j-1}\right)$

$\lambda_{j}=\sum_{k=1}^{j} \bar{D}_{k}^{\prime \prime}$ 
$j=1,2,3, \ldots, n, r \equiv O, E, U, V$

\section{Determination of future pattern swing direction}

Variation patterns in this work are generally considered to swing along increasing and decreasing directions. Increasing and decreasing pattern swing directions can be positive or negative at any time depending on current opening pattern swing properties. What is important to note is that while some patterns may swing in a certain direction, others may produce reverse swings. On the basis of this understanding, VPCPSs were then grouped according to the similarity in their ability to swing in the same direction given their presence in data. For example, if the most current swing is in the positive direction and favours an escalating pattern, then if a closing-lag pattern is anticipated as an expected future occurrence, its swing polarity must be in the direction opposite to the escalating pattern and its magnitude must result in a decrease in the current CVSM. Table 1 shows the grouping of pattern swings according to the effect of their swing values on the CVSM.

The expected future pattern swing values and directions are dependent on the magnitude and direction of the current $\operatorname{CVSM}\left(\lambda_{\text {cur }}\right)$, the current cumulative pattern swing(CPS) value $P_{\text {cur }}$ and pattern swing impulses $r_{\text {cur }}^{I}, r_{\min }^{I}$ and $r_{\max }^{I}$. $\lambda_{\text {cur }}^{I}$ and $P_{\text {cur }}$ were, respectively, obtained as

$\lambda_{\text {cur }}=\sum_{j=1}^{n} \bar{D}_{k}^{\prime \prime}$

$P_{\text {cur }}=\sum_{j=n}^{p} r_{j}^{L} \quad\left\{F\left\{r_{n}^{L}\right\}=1, \rho\left(r_{j}^{L}\right) \leftrightarrow \rho\left(r_{j-1}^{L}\right)\right\}$

$p=j \quad\left\{\rho\left(r_{j}^{L}\right) \uparrow \rho\left(r_{j+1}^{L}\right)\right\}$

$\lambda_{\text {cur }}$ values are given primary consideration in the determination of future variations swings. When $\lambda_{\text {cur }}=0$, then a future open pattern swing is expected. $P_{\text {cur }}$ and related impulses are employed for future variation swing determination analysis when $\lambda_{\text {cur }} \neq 0$.

$P_{\text {cur }}$ is used in determining the pre-expected future pattern swing direction for each pattern $\rho^{i}\left(r_{f}\right)$ (Table 2).

Table 1 VPCPSs grouped according to similarities in swing magnitude

\begin{tabular}{ll}
\hline Patterns $(r)$ & Expected swing magnitude \\
$O^{L}, E^{L}$ & Increasing absolute CVSM \\
$U^{L}, C^{L}$ & Decreasing absolute CVSM \\
$V^{L}$ & Decrease to zero, then increasing absolute CVSM \\
\hline
\end{tabular}

For example, given $P_{\text {cur }}\left(E_{\text {cur }}^{L}\right)$, that is, the current CPS being an escalation, if $\rho^{+}\left(P_{\text {cur }}\right)$ is the existing current swing direction, then, the pre-expected future pattern direction for a closure-lag $U_{f}^{L}$ will be the reversed polarity of the former.

$P_{\text {cur }}$ can only exist for a single VPCSPs $\left[r_{\text {cur }}^{L}(r: O \oplus E \oplus C \oplus U \oplus V)\right]$. However, a situation can occur in which $C_{n}^{L}$ and $V_{n}^{L}$ may exist within that same period due to their overlapping characteristics. In such situations, a preference to obtain $P_{\text {cur }}$ and $\rho\left(P_{\text {cur }}\right)$ from $V_{j}^{L}$ is usually made.

Pattern swing impulses and related parameters are used in the detecting expected future pattern swings. It is the final stage of the future pattern swing determination. These parameters are obtained from adjusting pattern swing values. The procedures for obtaining them differ from one VPCPS to another. The next section is devoted to discussing this.

\section{Pattern swing magnitude adjustments}

A rigorous adjustment process was employed in preparing pattern swing values for future swing estimation. Two adjustment procedures were employed. One procedure was carried out on the basis of CPS impulse and magnitude of occurrence, while the other was undertaken on the basis of the most frequently occurring pattern swings. The two procedures are presented next.

SDR parametric estimation and adjustment based on current and maximum cumulative swings After the split of $\bar{D}_{J}^{\prime \prime}$ into VPCPSs, parameters related to the duration of swings, recognised as being important for SDR future value analysis were obtained. These parameters are the current CPS impulses for escalation $E_{\text {cur }}^{I}$ and closure-lag $U_{\text {cur }}^{I}$, the maximum and minimum variation pattern swing impulses for escalation $\left(E_{\max }^{I}, E_{\min }^{I}\right)$, exact-closure

Table 2 VPCPSs grouped to show future swing direction given current swing

\section{Direction}

Current CPS $P_{\text {cur }}\left(r_{j}^{L}\right) \quad$ Expected future swing direction $\rho^{i}\left(r_{f}^{L}\right)$

\begin{tabular}{ll}
\hline$P_{\text {cur }}\left(r_{j}^{L}\right)\{r: O, E, V\}$ & $\rho\left(r_{f}^{L}\right) \leftrightarrow \rho\left(P_{\text {cur }}\right)\{r: O, E, V\}$ \\
$P_{\text {cur }}\left(r_{j}^{L}\right)\{r: O, E, V\}$ & $\rho\left(r_{f}^{L}\right) \uparrow \rho\left(P_{\text {cur }}\right)\{r: U, C\}$ \\
$P_{\text {cur }}\left(r_{j}^{L}\right)\{r: O, C\}$ & $\rho\left(r_{f}^{L}\right) \leftrightarrow \rho\left(P_{\text {cur }}\right)\{r: U, C\}$ \\
$P_{\text {cur }}\left(r_{j}^{L}\right)\{r: O, E, V\}$ & $\rho\left(r_{f}^{L}\right) \uparrow \rho\left(P_{\text {cur }}\right)\{r: O, E, V\}$
\end{tabular}


$\left(C_{\max }^{I}, C_{\min }^{I}\right)$ and closure-lag $\left(U_{\max }^{I}, U_{\min }^{I}\right)$ properties. The relations employed for obtaining these are provided in "Appendix B".

In addition, parameters related to actual current and current equivalent cumulative pattern swing magnitudes $E_{\text {cur }}^{* L}, E_{\text {cur }}^{\theta L}, U_{\text {cur }}^{* L}$ and $U_{\text {cur }}^{\theta L}$ were also determined. Before these were achieved $E_{j}^{L}$ and $U_{j}^{L}$ were adjusted to become $E_{j}^{* L}$ and $U_{j}^{* L}$. This was based on the understanding that due to the fuzzy nature of the variation properties, there exists the tendency for escalation and closure-lag properties to overlap and potentially occur within respective open and closing swings. These properties were identified and extracted from the respective mother set patterns into their respective subsets. The relations for achieving this are presented in "Appendix C". The current and current equivalent cumulative swing magnitudes for the adjusted escalating and closing lag swings were subsequently estimated ("Appendix D”).

Furthermore, there was also the need to update the respective VPCPSs values to reflect their magnitudes in the current period. The update was carried out as follows:

$$
\begin{aligned}
& r_{j}^{L \theta}=\left\{\begin{array}{l}
\max \left(\left|r_{j}^{L}\right|\right)\left\{\left|\bar{D}_{j}^{\prime \prime}\right|>\left|r_{j}^{L}\right|\right\} \\
\left|\bar{D}_{j}^{\prime \prime}\right|
\end{array}\left\{r:=O, E^{*}, C, U^{*}\right\}\right. \\
& V_{j}^{L \theta}=\left\{\begin{array}{l}
\max \left(\left|O_{j}^{L}+V_{j}^{L}\right|\right)\left\{\left|\bar{D}_{j}^{\prime \prime}\right|>\left|O_{j}^{L}+V_{j}^{L}\right|\right\} \\
\left|\bar{D}_{j}^{\prime \prime}\right|
\end{array}\right.
\end{aligned}
$$

With all the estimated parameters and adjustments made, future variation pattern swings were expected to occur based on the set of logical rules presented in Table 3.

\section{Adjustments based on most frequently occurring swing values}

All pattern swing value adjustments made on the basis of their swing value frequencies $\left(r_{J}^{\phi}\right)$ require a similar procedure. However, determining $O_{J}^{\phi}$ demands a slightly modified procedure. The steps required for obtaining, $E_{f}^{\phi}, C_{f}^{\phi}, U_{f}^{\phi}$ and $V_{f}^{\phi}$ are outlined below followed by the modified form of the procedure developed for obtaining $O_{j}^{\phi}$.

a. Procedure for obtaining any of $E_{f}^{\phi}, C_{f}^{\phi}, U_{f}^{\phi}$ and $V_{f}^{\phi}$ Step1: identify $\rho^{i}\left(r_{f}^{L}\right)$.

Step 2: employ $\rho^{i}\left(r_{f}^{L}\right)$ in obtaining $r_{\text {max }}^{L}$

$r_{\max }^{L}=\left\{\begin{array}{ll}\max \left(r_{j}^{L \beta}\right) & \left\{r_{j}^{L \beta}>0, \rho^{+}\left(r_{f}^{L}\right)\right\} \\ -\left(\max \left(\left|r_{j}^{L \beta}\right|\right)\right) & \left\{r_{j}^{L \beta}<0, \rho^{-}\left(r_{f}^{L}\right)\right\}\end{array}\right.$.

Step 3: replace all $r_{j}^{L \beta}$ values having polarities in reverse of $\rho^{i}\left(r_{f}^{L}\right)$ and convert data into absolute values.

$r_{j}^{* L \beta}=\left\{\begin{array}{ll}\left|r_{\max }^{L}\right| & \left\{\rho\left(r_{j}^{L \beta}\right) \uparrow \rho\left(r_{f}^{L}\right)\right\} \\ \left|r_{j}^{L \beta}\right| & \left\{\rho\left(r_{j}^{L \beta}\right) \uparrow \rho\left(r_{f}^{L}\right)\right\}\end{array}\right.$.

Step 4: identify the values of $r_{j}^{* L \beta}$ which account for $75 \%$ of the set on the basis of the most frequent swing magnitude values. Let the set for which the required data exist be $\{S 75\}$ and $b_{i}\left\{i=1, \ldots, i^{*}\right\}$ be the members of $\{S 75\}$.

Step 5: update $r_{j}^{* L \beta}$ to eliminate swing values that do not belong in $\{S 75\}$

$r_{j}^{\phi}=\left\{\begin{array}{ll}r_{j}^{* L \beta} & \left\{r_{j}^{* L \beta} \in\{S 75\}\right\} \\ {\left[b_{i} \min \left(\left|r_{j}^{* L \beta}-b_{i}\right|\right)\right]} & \text { otherwise }\}\end{array}\right.$.

Step 6: determination of future pattern swing.

This is the final stage of this procedure. The techniques developed and used for estimating $r_{j}^{L}$ is discussed in the next subsection.

b. Procedure for obtaining $O_{j}^{\phi}$

Notice from Table 3 that when the swing existing in the most current period results in a cumulative swing magnitude of zero, then the expected future swing automatically becomes an open swing. In such a situation, the implication is that there is no certainty on the direction of the next variation in swing. In respect of this, step 1 of the above
Table 3 Logical rules guiding type of future variation pattern occurrence

\begin{tabular}{ll}
\hline Expected future pattern occurrence & Conditions favouring pattern occurrence \\
\hline$O_{f}^{L}$ & $\lambda_{\text {cur }}=0$ \\
$E_{f}^{L}$ & $\lambda_{\text {cur }} \neq 0 ; A ;\left(E_{\text {cur }}^{I}+1 \leq E_{\text {max }}^{I}\right) \oplus\left(E_{\text {cur }}^{\theta L}>\left|E_{\text {cur }}^{* L}\right|\right)$ \\
$C_{f}^{L}, U_{f}^{L}, V_{f}^{L}$ & $\lambda_{\text {cur }} \neq 0 ; A ; B$ \\
$U_{f}^{L}$ & $\lambda_{\text {cur }} \neq 0 ; A ;\left(U_{\text {cur }}^{I}+1 \leq U_{\max }^{I}\right) \oplus\left(U_{\text {cur }}^{\theta L}>\left|U_{\text {cur }}^{* L}\right|\right)$ \\
$A \equiv \exists r: F\left(r_{j}^{L}\right)=1\left\{\rho\left(r_{j}^{L}\right) \leftrightarrow \rho\left(r_{f}^{L}\right)\right\}(r:=O, E, C, V, U)$ \\
$B \equiv C_{\min }^{I}-1 \geq 0\left\{C_{\min }^{I} \neq 0\right\}$
\end{tabular}


adjustment procedure loses its relevance. Determination of $r_{\max }^{L}$ in step 2 is modified thus,

$O_{\max }^{L}=\max \left(\left|O_{j}^{L \beta}\right|\right)$

Step 3 to step 6 of the previous procedure is maintained and applied as previously discussed.

\section{Future pattern estimation using decomposed state transition approach (DSTA)}

The DSTA involves the creation of various transition patterns. If any of the created patterns are dominant within $r_{j}^{\phi}$ then it is most likely that $r_{\text {cur }}^{\phi}$ will transit from its current state to a future state $r_{f}^{L}$ via the dominant pattern.

Four different pattern transition techniques were developed for this purpose. The techniques are (i) same state pattern switch, SSPS $(\alpha 1)$; (ii) cross state pattern switches CSPS $(\alpha 2)$; (iii) pattern span measure, PSM ( $\alpha 3$ ), and (iv) static dominant patterns, SDP $(\alpha 4)$. Only one of the first three techniques can be employed during any SDR analysis, while $\alpha 4$ is an inclusive technique adopted for detecting overwhelming static pattern occurrences that cannot be detected by the other three. We proceed to discuss how the methods are developed, the procedure employed in choosing the most appropriate technique for determining $r_{f}^{L}$ and the application of the chosen technique.

Application of the concept of Markov transition in developing the DSTA

The four future VPCPS determination techniques were derived by investigating series of swing patterns chains produced by periodic changes in $r_{j}^{\phi}$ values. Let $r_{j}^{\phi}$ exist as a Markov chain. Also, let $c_{j, j+1}$ be the link chain produced by $r_{j}^{\phi} \rightarrow r_{j+1}^{\phi}$, while $c_{j, j+1} \rightarrow c_{\text {cur-1,cur }}$ is the link chain produced by $r_{j}^{\phi} \rightarrow r_{j+1}^{\phi} \rightarrow r_{j+2}^{\phi} \rightarrow \cdots \rightarrow r_{\text {curr }}^{\phi}$. With respect to the future swing pattern estimation techniques, the following link chains were considered in this study:

$c_{j, j+1} \rightarrow c_{j, j+2}$

\{If investigating for $\alpha 1$ and $\alpha 2 ; j=1,2,3, \ldots, n-3\}$

$c_{j, j+1}$

$\{$ If investigating for $\alpha 3$ and $\alpha 4 ; j=1,2,3, \ldots, n-2\}$

Each periodic link chain investigated revealed a type of swing pattern existing within it subject to certain confirmatory conditions. The frequency of swing pattern occurrence $F^{d}$ within each link was subsequently computed for the four techniques developed. Finally, the probability of
VPCPS transition from its most current period to the future period was determined by computing the pattern occurrence strength index $I^{d}$ using the link chain analysis. The developed pattern recognition methods are fuzzified component elements of the Markov transition technique and function by determining the next transition state from the previous (only a single step transition was considered in this work). The methods and procedure for achieving this are outlined next.

Methods and procedure for obtaining the most dominant $r_{j}^{\phi}$ transition technique

1. Split $r_{j}^{\phi}$ into two new sets $W^{a}$ and $W^{b}$

$r_{j}^{\phi} \in \begin{cases}W^{a} & \left\{\left(r_{j}^{\phi}=1\right) \oplus\left(r_{j}^{\phi}=2\right)\right\} \\ W^{b} & \text { \{otherwise }\}\end{cases}$

Inferences were obtained using $r_{j}^{\phi}, W^{a}$ and $W^{b}$

2. Investigate the order of pattern swing occurrence,

$$
\begin{aligned}
& r_{j}^{\phi} \rightarrow r_{j+1}^{\phi} \rightarrow r_{j+2}^{\phi} \\
& \quad\{\text { for } \alpha 1 \text { and } \alpha 2 ; j=1,2,3, \ldots, n-3\} \\
& r_{j}^{\phi} \rightarrow r_{j+1}^{\phi}\{\text { for } \alpha 3 \text { and } \alpha 24 ; j=1,2,3, \ldots, n-2\}
\end{aligned}
$$

3. Obtain the frequency of occurrences $F^{d}$ for all techniques $d$.

$$
\begin{aligned}
& F^{d}=\sum_{h=1}^{h *} \tau^{d}\{h\} \\
& \tau^{d}\{h\}=1 \\
& h h=1,2, \ldots, h^{*}
\end{aligned}
$$

Table 4 Conditions necessary for obtaining order of pattern occurrences frequencies

\begin{tabular}{ll}
\hline $\begin{array}{l}\text { Desired pattern } \\
\text { occurrence } \\
\text { frequency }\end{array}$ & Necessary conditions \\
\hline$\tau^{\alpha . \alpha}(h)$ & $r_{j}^{\phi}, r_{j+1}^{\phi}, r_{j+2}^{\phi} \in W^{a}, r_{j}^{\phi} \neq r_{j+1}^{\phi}, r_{j+1}^{\phi} \neq r_{j+2}^{\phi}$ \\
$\tau^{\alpha .1 a}(h)$ & $r_{j}^{\phi}, r_{j+1}^{\phi}, r_{j+2}^{\phi} \in W^{b}, r_{j}^{\phi} \neq r_{j+1}^{\phi}, r_{j+1}^{\phi} \neq r_{j+2}^{\phi}$ \\
$\tau^{\alpha .2}(h)$ & $\left(r_{j}^{\phi} \in W^{b}\right) \oplus\left(r_{j}^{\phi} \in W^{a}\right),\left(r_{j+1}^{\phi} \in W^{a}\right)$ \\
& $\oplus\left(r_{j+1}^{\phi} \in W^{b}\right),\left(r_{j+2}^{\phi} \in W^{b}\right) \oplus\left(r_{j+2}^{\phi} \in W^{a}\right)$ \\
$\tau^{\alpha 3}(h)$ & $r_{j}^{\phi} \neq r_{j+1}^{\phi}$ \\
$\tau^{\alpha .4 a}(h)$ & $r_{j}^{\phi}=r_{j+1}^{\phi} ; r_{j}^{\phi}, r_{j+1}^{\phi} \in W^{a}$ \\
$\tau^{\alpha .4 b}(h)$ & $r_{j}^{\phi}=r_{j+1}^{\phi} ; r_{j}^{\phi}, r_{j+1}^{\phi} \in W^{b}$ \\
\hline
\end{tabular}




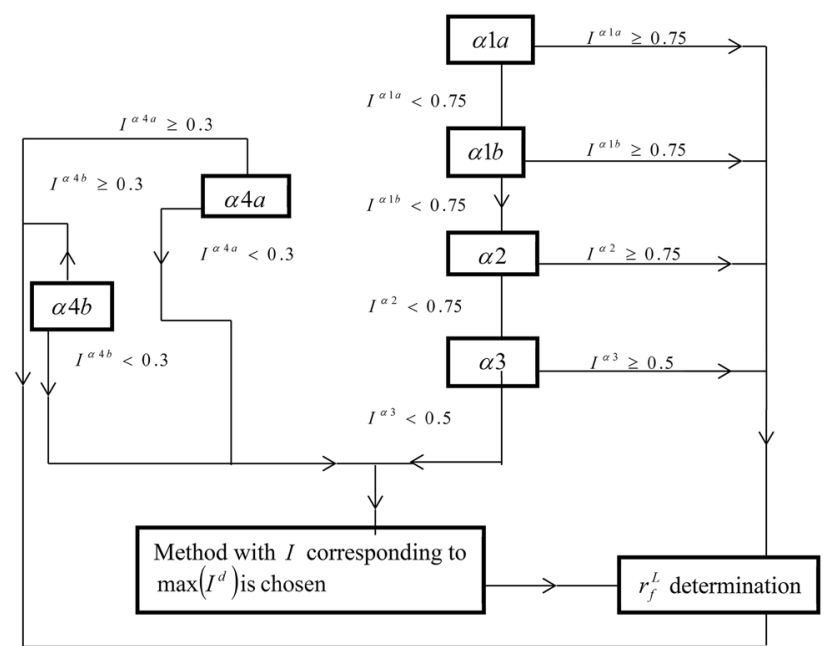

Fig. 3 Chart for obtaining the most appropriate technique for future pattern determination

represents the terminal points of the pattern swing chain investigated for which the conditions necessary for $\tau^{d}\{h\}$ to exist are fulfilled. These conditions are presented in Table 4.

4. Determine pattern occurrence strength index $\left(I^{d}\right)$

$$
I^{d}= \begin{cases}F^{d} / n-2 & \{d: \alpha 3, \alpha 4 a, \alpha 4 b\} \\ F^{d} / n-3 & \{d: \alpha 1 a, \alpha 1 b, \alpha 2\}\end{cases}
$$

$\alpha 1, \alpha 2$, and $\alpha 3$ were set in order of preference such that if any of its equivalent $I^{d} \geq 0.75$, then such a technique is considered the most appropriate for determining $r_{f}^{L}$, while other techniques not yet explored are ignored. Figure 3 is a chart which shows this order of preference

\section{Determination of $r_{f}^{L}$}

At the end of the future pattern swing value estimation technique determination procedure, given that the desired pattern technique(s) had been detected, the following relations were subsequently employed to obtain $r_{f}^{L}$.

1. SSPS

$$
r_{f}^{L}(q)=\left\{\begin{array}{ll}
A & \left\{r_{n}^{\phi}(q)=B\right. \\
B & \left\{r_{n}^{\phi}(q)=A\right.
\end{array}\right\}\left\{A, B \in W^{q} ; I^{\alpha 1 q} \geq 0.75\right\}
$$

\section{CSPS}

$$
r_{f}^{L}\{q, g\}=\left\{\begin{array}{l}
A_{g} \quad\left\{B_{g}, r_{n}^{\phi} \in W^{q} ; A_{g} \notin W^{q}\right\} \\
B_{g}\left\{A_{g}, r_{n}^{\phi} \in W^{q} ; B_{g} \notin W^{q}\right\}
\end{array}\left\{I^{\alpha 2 q} \geq 0.75\right\}\right.
$$

3. SDP

$r_{f}^{L}\{q, g\}=A_{g}\left\{I^{\alpha .4 q}\left\{A_{g}\right\} \geq 0.3 ; A_{g} \in W^{q}\right\}$

$q:=a, b ; g:=$ all pattern swing values of different magnitudes belonging to set $W^{q}$

4. PSM

$r_{f}^{L}$ determination by PMS involved some steps. First, the weighted mean ratio of $r_{j}^{\phi}$ occurrence was obtained.

$$
\bar{\alpha} 3=\left(\sum_{j=1}^{n-2} j\right)^{-1} \sum_{j=1}^{n-2} j\left(\max \left(r_{j}^{\phi}, r_{j+1}^{\phi}\right) / \min \left(r_{j}^{\phi}, r_{j+1}^{\phi}\right)\right)
$$

Employing $\bar{\alpha} 3$ two possible $r_{f}^{L}$ integer values $A$ and $B$ will exist. That is

$A=r_{n}^{\phi} / \bar{\alpha} 3$
$B=r_{n}^{\phi} \times \bar{\alpha} 3$

From these

$r_{f}^{L}= \begin{cases}A & \{A \in R ; B \notin R\} \\ B & \{B \in R ; A \notin R\}\end{cases}$

$R$ is the interval bound by $\left[\min \left(r_{j}^{\phi}\right), \max \left(r_{j}^{\phi}\right)\right]$.

Situations in which $A, B \in R$ were also observed to exist. In such cases, five rules were created and used in obtaining $r_{f}^{L}$ from $A$ and $B$. The rules and relations for obtaining future pattern values are presented in "Appendix E".

Having obtained $r_{f}^{L}(i)\left\{i=1, \ldots, i^{*}: 1 \leq i^{*} \leq n-1\right\}$, two situations of note is pointed out.

1. The analysis just carried out concerns situations of $\rho^{+}\left(r_{f}^{L}\right)$. For the situations involving $\rho^{-}\left(r_{f}^{L}\right)$, all $r_{f}^{L}(i)$ obtained was converted to their negative equivalent.

2. Recall that in situations $\lambda_{\text {cur }}=0$ the predictability of the next direction of swing cannot be out rightly ascertained in this work. To account for this degree of uncertainty, the future pattern swing $O_{f}$ at this stage was analysed in both the forward and backward direction. Thus, the future values for an expected open swing became the union of its future values in the positive and negative directions.

$O_{f}^{* L}=\left\{O_{f}^{L},-O_{f}^{L}\right\}$

\section{Verification expected future pattern swing values}

Two activities were adopted in assessing $r_{f}^{L}(i)$ to control and reduce overestimation of SDR forecasts. The activities involve verification to establish maximum pattern swing conformity and conformation to swing time packet limits. 
1. Verification of $r_{f}^{L}(i)$ for maximum pattern swing conformity

This activity involves a comparison of $r_{f}^{L}(i)$ values against $r_{\text {max }}^{L}$. This comparison was necessitated by the need to ensure that $r_{f}^{L}(i)$ does not exceed its maximum allowable swing.

$r_{f}^{L}(i)= \begin{cases}r_{\max }^{L} & \left\{\left|r_{f}^{L}(i)\right|>\left|r_{\max }^{L}\right| ; i=1, \ldots, i^{*} ; r=O^{*}, E, U, V\right\} \\ r_{f}^{L}(i) & \{\text { otherwise }\}\end{cases}$

2. Verification of $r_{f}^{L}(i)$ for pattern swing time packet limit conformity

This verification procedure was undertaken on the outcome of the first stage verification. Here, $r_{f}^{L}(i)$ values were compared against currently existing variation characteristics to ensure that they did not exceed the existing maximum time packet value limits. To achieve this, there was a need to revert $\lambda_{\text {cur }}$ to its time packet values. Let $\chi_{z}$ be a vector in which the time packet values exist. Then the break-up of $\lambda_{\text {cur }}$ into its time packet components $\chi_{z}$ is expressed as:

$$
\begin{aligned}
& \chi_{z}=G_{t} \quad\left\{z=1,2, \ldots, z^{*} ; t=t^{*}, t^{*}-1, \ldots, 1\right\} \\
& G_{t}=\left\{\begin{array}{cc}
\bar{D}_{j} & \left\{\left|\lambda_{t-1}^{*}\right|>\left|\bar{D}_{j}\right| ; \rho\left(\lambda_{t-1}^{*}\right) \leftrightarrow \rho\left(\bar{D}_{j}\right)\right\} \\
\lambda_{t-1}^{*} & \left\{\left|\lambda_{t-1}^{*}\right| \leq\left|\bar{D}_{j}\right| ; \rho\left(\lambda_{t-1}^{*}\right) \leftrightarrow \rho\left(\bar{D}_{j}\right)\right\}
\end{array}\right. \\
& \lambda_{t}^{*}=\lambda_{t-1}^{*}-G_{t} \quad\left\{\left|\lambda_{t-1}^{*}\right|>\left|G_{t}\right|\right\} \\
& \lambda_{0}^{*}=\lambda_{\text {cur }} \\
& t=1,2,3 \ldots, t^{*}\left[t^{*}=t\left(G_{t}=\lambda_{t-1}^{*}\right)\right], \\
& \quad j=1,2,3, \ldots, j^{*}\left[j\left(G_{t}=\lambda_{t-1}^{*}\right)\right]
\end{aligned}
$$

The time packet value limit verification process is unique to different VPCPS type. "Appendix F" presents the relations necessary for verifying $r_{f}^{\mathcal{L}}(i)$ to become $r_{f}^{* L}(i)$. This second stage verification is not applicable to future patterns obtained from expected open pattern swings.

\section{SDR reversal, defuzzification and GFMAPR forecast span generation}

At the end of the SDR fuzzification and future pattern determination analysis, $r_{f}^{* L}(i)$ were reversed and used in generating GFMAPR forecast intervals. This section undertakes a discussion on the interval generation procedure which was carried out in three phases.

Phase1: SDR reversal

The reversal process involved the declassification of the SLV classes into their component fuzzy variation states.
Let $Z$ be a set consisting of the union of $r_{f}^{* L}(i)\{r:=O, E, U, V\}$, having elements $q^{*}$

$q^{*}=\# Z$

$\delta_{b(j)}^{f}=\left\{\begin{array}{c}\delta_{i, i+1} \\ -\delta_{i, i+1}\end{array} \quad\left\{\begin{array}{l}\left.Z_{q}=\bar{D}_{j}, \delta_{i, i+1} \in \bar{D}_{j}, \rho^{+}\left(Z_{q}\right)\right\} \\ \left.Z_{q}=\left|\bar{D}_{j}\right|,\left|\delta_{i, i+1}\right| \in \bar{D}_{j}, \rho^{-}\left(Z_{q}\right)\right\}\end{array}\right.\right.$

$\left\{j=1,2,3,4 ; i=1,2, \ldots, n-1 ; q=1,2, \ldots, q^{*} ;\right.$

$$
\left.b=1,2,3, \ldots, b^{*}\left(b^{*}=i q\right)\right\}
$$

Phase 2: Determination of fuzzified forecast states and subsequent defuzzification

Employing the forecast SLV span $\delta_{b}^{f}$ $\left\{b=1,2,3, \ldots, \sum_{j=1}^{4} b(j)\right\}$ the forecast FLV state numbers $k_{b}^{f}$ were subsequently obtained as:

$k_{b}^{f}=k\left(s_{\text {cur }}^{\prime}=s_{k}\right)+\delta_{b}^{f}$

$b$ number of SDR forecast intervals were obtained or generated (as the case may be) by locating the FLV forecast state $s_{b}^{f}$ using $k_{b}^{f}$. The forecast FLV states $s_{b}^{f}$ or $\left(s_{b}^{f[L B]}, s_{b}^{f[U B]}\right)$ was then obtained from the $\delta_{b}^{f}$ as:

$$
\left(s_{b}^{f[L B]}, s_{b}^{f[u B]}\right):= \begin{cases}\left(s_{\text {cur }}^{\prime L}+\omega \Delta k, s_{\text {cur }}^{\prime U}+\omega \Delta k\right. & \left\{\rho^{+}\left(\delta_{b}^{f}\right)\right\} \\ \left(s_{\text {cur }}^{\prime L}-\omega \Delta k, s_{\text {cur }}^{\prime U}-\omega \Delta k\right. & \left\{\rho^{-}\left(\delta_{b}^{f}\right)\right\}\end{cases}
$$

The fuzzy FLV states were then de-fuzzified into actual forecast intervals $\hat{x}_{b}^{f}$ using a modified form of Eq. (22), the interval bounds were obtained as:

$\hat{x}_{b}^{f[L B]}=s_{B}^{f[L B]}-\bar{s}_{\text {cur }}^{\prime}+\hat{x}_{n}$

$\hat{x}_{b}^{f[U B]}=s_{B}^{f[U B]}-\bar{s}_{\text {cur }}^{\prime}+\hat{x}_{n}$

Phase 3: SDR forecast interval generation

This phase is an extension of the second phase. It considers two major characteristics of industrial accident occurrences. These are steady trends and fluctuations. GFMAPR was created from two interval variants which give consideration to these characteristics. The two interval variants are the unbound generated interval (UBGInt) and the bound generated interval (BGInt). Equations (77) and (78) are employed for generating the intervals for both variants. Prior to obtaining BGInt some adjustments are made to $\hat{x}_{b}^{f}$ such that,

$$
\begin{array}{ll}
s_{b}^{f[L B]}=s_{1} & \left\{s_{b}^{f[L B]}<s_{1}\right\} \\
s_{b}^{f[U B]}=s^{*} & \left\{s_{b}^{f[U B]}>s^{*}\right\}
\end{array}
$$


Notice that BGInt limits forecast strictly to the initial universe of discourse. The use of BGInt for GFMAPR prediction will strongly favour fluctuating situations, while UBGint is created to adapt well to trend occurrences where future values are expected to exist outside a non-preempted universe of discourse. A combination of these two adaptations makes up the GFMAPR.

\section{Procedure for the multiplicative data relationship determination}

The SDR future value predictions are intervals values and thus cannot be unitarily employed to obtain point value forecasts. To be able to determine actual accident forecasts, a complementary approach which is the MDR is also developed. The MDR employs a procedure somewhat similar to the SDR but the method differs. The discussion of the procedure is carried out in relation to the outline in Fig. 1.

\section{MDR preparation}

The input data for the MDR were obtained from indices resulting from a comparative variation analysis of historical data.

$z_{i}^{\circ}=x_{i+1} / x_{i}\{i=1,2,3, \ldots, n-1\}$

Based on the above, the width of change in variation is expressed as

$$
\begin{aligned}
\omega^{\circ}= & \begin{cases}\min \left(\Omega^{\Phi}, \Omega^{\wedge}\right) & \left\{\Omega^{\Phi}, \Omega^{\wedge} \neq 0\right\} \\
\max \left(\Omega^{\Phi}, \Omega^{\wedge}\right) & \left\{\left\{\Omega^{\Phi}=0\right\} \oplus\left(\Omega^{\wedge}=0\right)\right\} \\
\min \left(H_{b}^{\wedge}\right) & \text { otherwise }\}\end{cases} \\
\Omega^{m}= & \sum_{j=1}^{Q(m)-1} \sum_{t=j+1}^{Q(m)}\left(\left|H_{j}^{m}-H_{t}^{m}\right|\right) \\
& / 2 \sum_{j=1}^{Q(m)-1} \sum(Q(m)-j)_{j} \quad\{j=1,2,3, \ldots, Q(m)-1\}
\end{aligned}
$$

$H_{a}^{\Omega}=Z_{i}^{\circ} \quad\left\{Z_{i}^{\circ}>1\right\}$

$H_{b}^{\wedge}=z_{i}^{\circ} \quad\left\{z_{i}^{\circ} \leq 1\right\}$

$\{m: \Phi, \wedge ; Q(\Phi)+Q(\wedge)=n-1\}$

$s_{k}^{\circ}$ was then obtained using expression (17) with $z_{i}$ and $\omega$ replaced by $z_{i}^{\circ}$ and $\omega^{\circ}\{i=1,2,3, \ldots, n-1\}$, respectively. Note that the boundary adjustment for the SDR initial universe of discuss (expression (18)) is not applicable here. With $z_{i}$ and $a_{k}$ duly replaced in relations (19) and (20), fuzzification and location of $z_{i}^{\circ}$ in $s_{k}^{\circ}$ was also undertaken.
MDR second level variations $\delta_{i, i+1}^{\circ}\{i=1,2,3, \ldots, n-2\}$ and fuzzy class and representations $\bar{D}_{j}^{\circ}$ were also obtained using relations (23)-(30).

With respect to $\bar{D}_{j}^{\circ}$, an exceptional consideration was given to situations where, $n=4 \quad \# \bar{D}_{j}^{\circ}=1$ and $\# \bar{D}_{j}^{\circ}=0\{j \neq 1\}$. In such circumstances, the single element in $\bar{D}_{j}^{\circ}$ with value $|e|$ was adjusted by creating other values between 1 and $|e|$ to exist as elements in $\bar{D}_{j}^{\circ}$ (expression (86)).

$\bar{D}_{j}^{* \circ}=\left\{\begin{array}{cc}1 \leq\left|e_{m}^{*}\right| \leq G_{j} & \{j=1\} \\ G_{j-1}<\left|e_{m}^{*}\right| \leq G_{j} & \{j>1\}\end{array}\right.$

$G_{j}=j \times(|e| / 4)$

$\left|e_{m}^{*}\right|$ are various elements in $\bar{D}_{j}^{* \circ}$ with the maximum number of elements in each set dictated by the conditions presented in relation (87). It should be noted that $\left|e_{m}^{*}\right|$ must exist as integers. Thus, decimal forms must be rounded up to their corresponding nearest integer values.

Creating this exceptional procedure was necessary because of the availability of a single variation packet having the possibility of a large variation space existing within it. Thus, we endeavoured to glean as much information as possible given such situations by exhaustively investigating the major regions within this variation space.

\section{Determination of MDR second-level variation future values}

This is undertaken in two steps, namely (i) investigation of $z_{i}^{\circ}$ to ascertain its most dominant variation characteristics and (ii) determination of future variation values on the basis of $z_{i}^{\circ}$ most dominant trend or fluctuating characteristics. Before proceeding, let $\beta_{i}^{\circ}=\delta_{i, i+1}^{\circ}\{i-1,2,3, \ldots, n-2\}$.

$\beta_{f}^{\circ T}$ is the set of MDR future value forecasts obtained from trend dominant $\beta_{t}^{\circ}$.

$\beta_{f}^{\circ F}$ is the set of MDR future value forecasts obtained from fluctuation dominant $\beta_{t}^{\circ}$.

\section{Determination of data variation characteristics}

Two major characteristics were investigated, namely trend and fluctuations. In estimating the degree of trending $\eta^{T}$, and fluctuating $\eta^{F}$ properties of $z_{i}^{\circ}$, the relation below was employed:

$\eta^{T}=N^{T} / N^{d}$

$\eta^{F}=1-\eta^{T}$

The quad-point data characteristic trace (Edem et al. 2016) was employed to determine the number of trend 
chains existing in $z_{i}^{\circ}\left(N^{T}\right)$ and the total number of $z_{i}^{\circ}$ quadpoints $\left(N^{d}\right) . \eta^{T}$ and $\eta^{F}$ were subsequently employed as $\beta_{i}^{\circ}$ control parameters for the determination $r_{f}\left\{r: \beta_{f}^{T}, \beta_{f}^{\circ F}\right\}$.

Future second-level variation value based on more dominant data variation characteristic

The procedure for obtaining $\beta_{f}^{\circ T}(q)$ differs from that used in obtaining $\beta_{f}^{\circ T}\left(q^{\prime}\right)$. Both procedures are discussed exclusively.

Procedure for determining $\beta_{f}^{\circ T}(q)$ If $\eta^{T} \geq 0.75$, then the following applies:

1. Determine from $\beta_{i}^{\circ}\{i=2, \ldots, n-3\}$ the most current trend chain $T_{w}^{\circ} \cdot\left\{w=1,2, \ldots, w^{*}\right\}$. This is achieved by obtaining $T_{t(y)}^{\circ}$ which are various sets of elements in $\beta_{i}^{\circ}$ having similar polarities.

$T_{t(y)}^{\circ}=\beta_{t-1}^{\circ} \quad\{A \oplus B\}$

$T_{t^{*}(y)}^{\circ}=\beta_{i}^{\circ} \quad\{B\}$

$A=\rho\left(\beta_{i-1}^{\circ} \leftrightarrow \rho\left(\beta_{i}^{\circ}\right) \leftrightarrow \rho\left(\beta_{i+1}^{\circ}\right)\right)$

$B=\rho\left(\beta_{i-1}^{\circ}\right) \leftrightarrow \rho\left(\beta_{i}^{\circ}\right) \uparrow \rho\left(\beta_{i+1}^{\circ}\right)$

$v_{i}=\left\{\begin{array}{cl}1 & \{i=2\} \\ y^{*}+1 & \text { \{otherwise }\}\end{array}\right.$

$y^{*}=y\left\{\rho\left(\beta_{i-1}^{\circ}\right) \leftrightarrow \rho\left(\beta_{i}^{\circ}\right) \uparrow \rho\left(\beta_{i+1}^{\circ}\right)\right\}$

$t(y)=1,2, \ldots, t^{*}(y) ; y=1,2 \ldots, y^{* *} ; 1<y^{* *} \leq n-1$

$t^{*}(y)=t(y)+1=\left\{\rho\left(\beta_{k-1}^{\circ}\right) \leftrightarrow \rho\left(\beta_{k}^{\circ}\right) \uparrow \rho\left(\beta_{k+1}^{\circ}\right)\right\}$
$T_{w}^{\circ}=T_{t(y=\max (\mathrm{y}))}^{\circ} \quad\left\{\# T_{t(y)}^{\circ}>0\right\}$

2. Investigate $T_{w}^{\circ}\left(w=1,2, \ldots, w^{*}-1\right)$ such that

$\beta_{f}^{T \circ}(q)=T_{w}^{\circ} \quad\left\{T_{w}^{\circ}=T_{w *}^{\circ}\right\}$

3. If $\# \beta_{f}^{T \circ}=0$ at the end of step 2, then apply the concept developed in Sect. 4.5.4 to obtain $\beta_{f}^{T \circ}(q)$.

Procedure for determining $\beta_{f}^{T \circ}$ values

The determination of $\beta_{f}^{T \circ}(q)$ from fluctuating variation characteristics involved a combination of two Markov transition based techniques. Before proceeding, three major required parameters are defined. Let $B_{i}^{*}$ be a dynamic universal interval of any period $i$ with the most positive bound $B_{i}^{*[L B]}$ and most negative bound $B_{i}^{*[U B]}$ for which all fluctuating transitions take place (no transition swing value can exceed $B_{i}^{*}$ ).

$B_{i}^{*}:=\left(B_{i}^{*[L B]}, B_{i}^{*[U B]}\right)$

The difference between $B_{i}^{*[L B]}$ and $B_{i}^{*[U B]}$ is always constant and equal to the variation sway magnitude $(\gamma)$.

$v$ : is the most current pattern swing variation position.

Given that $\eta^{T}<0.75$, analysis on $\beta_{i}^{\circ}$ was carried out to obtain $B_{n-2}^{*} \gamma_{n-2}$ and $v_{n-2}$ as a prerequisite to determining the future sway direction $\beta_{f}^{\circ F g}$. Equations (100), (101) and

Table 5 Various $B_{i}^{*}$ boundary for varying requisite parameter conditions

\begin{tabular}{llll}
\hline$B_{i}^{*[L B]}$ value & Conditions that favour $B_{i}^{*[L B]}$ value & $B_{i}^{*[U B]}$ value & Conditions that favour $B_{i}^{*[U B]}$ value \\
\hline 0 & $\rho^{+}\left(\beta_{1}^{\circ}\right), i=1$ & 0 & $\rho-\left(\beta_{1}^{\circ}\right), i=1$ \\
$\beta_{1}^{\circ}$ & $\rho-\left(\beta_{1}^{\circ}\right), i=1$ & $\beta_{1}^{\circ}$ & $\rho^{+}\left(\beta_{1}^{\circ}\right), i=1$ \\
$v_{i}$ & $v_{i}<B_{i-1}^{*[\mathcal{L} B]} ; i>1$ & $v_{i}$ & $v_{i}>B_{i-1}^{*[U B]} ; i>1$ \\
$B_{i-1}^{*[L B]}$ & $v_{i} \geq B_{i-1}^{*[\mathcal{L} B]} ;\left(B_{i}^{*[\mathcal{L} B]}-B_{i-1}^{*[\mathcal{L} B]}\right) \leq \gamma_{i} ; i>1$ & $B_{i-1}^{*[L B]}$ & $v_{i} \leq B_{i-1}^{*[\mathcal{U} B]} ;\left(B_{i}^{*[\mathcal{L} B]}-B_{i-1}^{*[\mathcal{L} B]}\right) \leq \gamma_{i} ; i>1$ \\
$B_{i}^{*[U B]}-\gamma i$ & $v_{i} \geq B_{i-1}^{*[\mathcal{L} B]} ;\left(B_{i}^{*[\mathcal{L} B]}-B_{i-1}^{*[\mathcal{L} B]}\right)>\gamma_{i} ;$ & $B_{i}^{*[L B]}-\gamma i$ & $v_{i} \leq B_{i-1}^{*[\mathcal{U} B]} ;\left(B_{i}^{*[\mathcal{U} B]}-B_{i-1}^{*[\mathcal{L} B]} \leq \gamma_{i} ;\right.$ \\
& $\rho^{-}\left(\beta_{i}\right) ; i>1$ & & $\rho^{+}\left(\beta_{i}\right) ; i>1$ \\
\hline
\end{tabular}

Table 6 Some industrial accidents data employed in the development of the GFMAPR search mechanism. Sources: Type 1: Jiang 2007; Type 2 and Type 3: Huang et al. (2012a, b)

\begin{tabular}{|c|c|c|c|c|c|c|c|c|c|c|c|c|}
\hline Type1 & 140280 & 142326 & 179955 & 189185 & 216784 & 258315 & 254811 & 252704 & & & & \\
\hline Type 2 & 217 & 571 & 674 & 847 & 992 & 925 & 990 & 812 & 918 & 961 & 876 & \\
\hline Type 3 & 108 & 81 & 87 & 59 & 69 & 55 & 67 & 56 & 58 & 77 & 82 & 113 \\
\hline
\end{tabular}


Table 5 show the relations used in obtaining the required requisite parameters (Table 6).

$v_{i}=\sum_{t=1}^{t} \beta_{t}^{\circ}$

$\gamma_{i}=\left\{\begin{array}{cl}\max \left(\left|\beta_{i}^{\circ}\right|\right) & \{\text { currently unsteady pattern } \\ \bar{\beta}^{\circ} & \{\text { currently steady pattern }\end{array}\right.$

$\bar{\beta}^{\circ}=\sum_{i=n-2}^{n-6} j(i) \times\left|\beta_{i}^{\circ}\right| / \sum_{i=n-2}^{n-6} j(i)$

$j(i)=\left\{\begin{array}{cc}5 & \{i=n-2\} \\ j(i+1)-1 & \text { \{otherwise }\}\end{array}\right.$

Determination of the steadiness in current pattern involved an investigation of $\beta_{1}^{\circ}\{i=n-2, n-3$, $\ldots, n-6\}$. If more than eighty percent of $\beta_{1}^{\circ}$ belong to exclusively to either the lower variation class $(1,2)$ or upper variation class $(3,4)$ then $\beta_{1}^{\circ}$ was adjudged to be currently steady, else the pattern was considered currently unsteady. If $i<5, \beta_{1}^{\circ}$ is also taken to be currently unsteady.

The technique for obtaining $B_{n-2}^{*}, \gamma_{n-2}$ and $v_{n-2}$ involved investigating $\beta_{1}^{\circ}$ from progressively from $i=1$ and steadily adjusting and obtaining $B_{i}^{*}, \gamma_{i}$ and $v_{i}$ until the point $i=n-2$ was attained. Based on the obtained parameters, $\beta_{f}^{\circ F g}\{g:=+,-\}$ were determined from the two techniques earlier mentioned.

a. First technique for $\beta_{f 1}^{\circ F g}$ determination

This method assumes that $v_{n-2}$ is a position within $B_{n-2}^{*}$. The distances between $v_{n-2}$ and $B_{n-2}^{*[L B]}$ as well as $v_{n-2}$ and $B_{n-2}^{*[U B]}$ represent the maximum transition magnitude of pattern swing variation from the most current position to the expected future position. Transitions made from $v$ can be made in the forward $\left(\beta_{f 1}^{\circ F+}\right)$, backward $\left(\beta_{f 1}^{\circ F-}\right)$ direction or in both directions. Transitions made from $v_{n-2}$ towards any ends of the universal bound are constrained or limited by $\gamma_{n-2} . \beta_{f 1}^{\circ F g}$ was obtained using the equation below:

$\beta_{f 1}^{\circ F g}=B_{n=2}^{*[M]}-v_{n-2}$

$M:=U B, L B, g:=+,-$

b. Second technique for $\beta_{f 2}^{\circ F g}$ determination

The second method gives stronger consideration to a highly fluctuating pattern in which $v_{n-2}$ does not exhibit huge deviation from $\gamma_{n-2} \cdot B_{n-2}^{*}$ was not given consideration in this method. $\beta_{f 2}^{\circ F g}$ values were obtained from the following relations: $\beta_{f 2}^{\circ F+}= \begin{cases}0 & \left\{\rho^{+}\left(v_{n-2}\right), v_{n-2}<\gamma_{n-2}\right\} \\ \gamma_{n-2}-v_{n-2} & \left\{\rho^{+}\left(v_{n-2}\right), v_{n-2} \geq \gamma_{n-2}\right\} \\ \gamma_{n-2}-\left|\beta_{f 2}^{\circ F-}\right| & \left\{\rho^{-}\left(v_{n-2}\right),\left|v_{n-2}\right|<\gamma_{n-2}\right\} \\ \gamma_{n-2} & \left\{\rho^{-}\left(v_{n-2}\right),\left|v_{n-2}\right| \geq \gamma_{n-2}\right\}\end{cases}$

$\beta_{f 2}^{\circ F+}= \begin{cases}0 & \left\{\rho^{-}\left(v_{n-2}\right),\left|v_{n-2}\right| \geq \gamma_{n-2}\right\} \\ \left|v_{n-2}\right|-\gamma_{n-2} & \left\{\rho^{-}\left(v_{n-2}\right),\left|v_{n-2}\right|<\gamma_{n-2}\right\} \\ \beta_{f 2}^{\circ F+}-\gamma_{n-2} & \left\{\rho^{+}\left(v_{n-2}\right), v_{n-2}<\gamma_{n-2}\right\} \\ -\gamma_{n-2} & \left\{\rho^{+}\left(v_{n-2}\right), v_{n-2} \geq \gamma_{n-2}\right\}\end{cases}$

The desired future forward and backward variation values were subsequently obtained as

$\beta_{f}^{\circ F+}=\max \left(\beta_{f 1}^{\circ F+}, \beta_{f 2}^{\circ F+}\right)$

$\beta_{f}^{\circ F-}=\min \left(\beta_{f 1}^{\circ F-}, \beta_{f 2}^{\circ F-}\right)$

The intention of carrying out the MDR analysis was to determine a region for which SDR forecast interval could intersect its forecast interval. As a result, $F_{f}^{\circ g}$ point values were considered in addition to all regions before it. Thus, the forward and backward future $M D R$ variation forecasts are expressed as

$\beta_{f}^{\circ F+}(h)\{h=1,2, \ldots, \beta\} \quad$ and

$\beta_{f}^{\circ F-}\left(h^{\prime}\right)\left\{h^{\prime}=-1,-2, \ldots, \beta_{f}^{\circ F-}\right\} \quad$ respectively.

$\beta_{f}^{\circ}\left(q^{\prime}\right)=\beta_{f}^{\circ F+}(h) \cup \beta_{f}^{\circ F-}\left(h^{\prime}\right)$

Before proceeding to discuss the defuzzification stage of the SDR, let $\beta_{f}^{\circ}\left(g^{\prime}\right)$ be the set which contains all MDR future variation values $\left\{\beta_{f}^{\circ}\left(g^{\prime}\right)=\beta_{f}^{T \circ}(q) \cup \beta_{f}^{\circ}\left(q^{\prime}\right) ; g^{\prime}=1,2,3, \ldots, q^{\prime} *\right\}$ $g^{\prime *}=q+q^{\prime}$

\section{Defuzzification procedure for the MDR}

At the end of the MDR future variation determination procedure, a defuzzification procedure involving a twostage process was carried out.

The determination of the future SLV values is the first process. This was achieved by identifying various $\delta_{i, i+1}^{\circ}$ values belonging to different $\bar{D}_{j}^{\circ}$ classes given that $\bar{D}_{j}^{\circ}$ or $-\bar{D}_{j}^{\circ}$ exist in $\beta_{f}^{\circ}\left(g^{\prime}\right)$. Point values of $\delta_{i, i+1}^{\circ}$ for each $\bar{D}_{j}^{\circ}$ found in $\beta_{f}^{\circ}(g)$ were then obtained using the weighted average technique.

$\delta_{j}^{* \circ f}=\left\{\begin{array}{cl}\delta_{j}^{\circ f} & \left\{\rho^{+}\left(\beta_{f}^{\circ}\left(g^{\prime}\right)\right)\right\} \\ -\delta_{j}^{\circ f} & \{\text { otherwise }\}\end{array}\right.$

where 
$\delta_{j}^{\circ f}=\sum_{i=1}^{n-2} i \delta_{i, i+1}^{\circ} / \sum_{i=1}^{n-2} i \quad\left\{\delta_{i, i+1}^{\circ} \in \bar{D}_{j}^{\circ}, \bar{D}_{j}^{\circ}=\left|\beta_{f}^{\circ}\left(g^{\prime}\right)\right|\right\}$

Recall that an exceptional fuzzification procedure was undertaken for situations involving $n=4$. The corresponding first-stage defuzzification relations are

$\delta_{j}^{* \circ f}= \begin{cases}e^{*} & \left\{e^{*} \in \bar{D}_{j}^{\circ}, \rho^{+}\left(\beta_{f}^{* L}\right), \# \bar{D}_{j}^{\circ}=1\right\} \\ -e^{*} & \left\{e^{*} \in \bar{D}_{j}^{\circ}, \rho^{-}\left(\beta_{f}^{* L}\right), \# \bar{D}_{j}^{\circ}=1\right\} \\ \left(\max \left(e_{m}^{*}\right)+\min \left(e_{m}^{*}\right)\right) / 2 & \left\{e_{m}^{*} \in \bar{D}_{j}^{\circ}, \rho^{+}\left(\beta_{f}^{* L}\right), \# \bar{D}_{j}^{\circ}>1\right\} \\ -\left(\max \left(e_{m}^{*}\right)+\min \left(e_{m}^{*}\right)\right) / 2 & \left\{e_{m}^{*} \in \bar{D}_{j}^{\circ}, \rho^{-}\left(\beta_{f}^{* L}\right), \# \bar{D}_{j}^{\circ}>1\right\}\end{cases}$

The second and final stage of the defuzzification procedure was the determination of the future first-level variation fuzzified state number $k_{j}^{\circ f}$ using $\delta_{j}^{* \circ}$.

$k_{j}^{\circ f}=k\left(s_{\mathrm{cur}}^{\prime^{\circ}}=s_{k}^{\circ}\right)+\delta_{j}^{* \circ f}$

Finally, defuzzified MDR FLV values $z_{j}^{o f}$ were obtained from corresponding $i_{j}^{\text {of }}$ as:

$z_{j}^{\circ f}=\bar{s}_{t}^{\circ}+\left[\left(\bar{s}_{t}^{\circ}-\bar{s}_{t+1}^{\circ}\right)\left(t-k_{j}^{\circ f}\right)\right]$

$t=k\left[\min \left(k-k_{j}^{\circ f}\right)\right] \quad\left\{k<k_{j}^{\circ f}\right\}$

$\left\{k=1,2,3, \ldots, k_{\max }\right\}$

where

$\bar{s}^{\circ}=\left(\bar{s}^{\circ L}+\bar{s}^{\circ U}\right) / 2$.

\section{GFMAPR forecasting from whitenisation of SDR and MDR future outcomes}

GFMAPR forecasting is achieved by a whitenisation process developed in this work. This involves the comparison and adoption of the intersection of grey SDR and MDR future forecast possibilities based on the satisfaction of a fixed intersection criterion. First, SDR future values are reformed to exist in the same orientation as MDR outcomes. This is treated in the first part of this section. The second part discusses the development of the intersection criterion. The final part of the section covers the use of the satisfied and unsatisfied criterion situations for undertaking GFMAPR forecasts.

\section{SDR point future values: creation and reformation}

It will be recalled that SDR future possibilities were obtained as a set of grey intervals $\hat{x}_{b}^{f}\left\{b=1,2,3, \ldots, b^{*}\right\}$ (Sect. 4.6). At this stage, each $\hat{x}_{b}^{f}$ was split into three point values namely $\hat{x}_{b}^{[L B]}, \hat{x}_{b}^{f U B]}$ and the mean value of the former two. Let $M_{d}^{f}\left\{d=1,2,3, \ldots, d^{*}\left(d^{*}=3 b^{*}-A\right)\right\}$ be the set in which the split values exist as elements provided that $\hat{x}_{b}^{[U B]} \neq \hat{x}_{b+1}^{[[L B]}$.

$A=\sum_{b=1}^{b^{*-1}} N_{b}\left\{\hat{x}_{b}^{f[U B]}=\hat{x}_{b+1}^{f[L B]}\right\}$

$N_{b}=1$

$M_{d}^{f}$ was subsequently reformed as $M_{d}^{* f}$ so that $M_{d}^{f}$ would have the same orientation as $z_{j}^{\circ f}$

$M_{d}^{* f}=M_{d}^{f} / x_{n}$

A comparison of the degree of deviation of $M_{d}^{* f}$ from $z_{j}^{\circ f}$ was then investigated.

$$
\begin{aligned}
& R_{j d}=z_{j}^{\circ f}-M_{d}^{* f} \\
& \quad\left\{j=1,2, \ldots, j *(1 \leq j * \leq 4) ; d=1,2,3, \ldots, d^{*}\right\}
\end{aligned}
$$

\section{Development of the forecast acceptability criterion (FAC)}

The purpose of determining $R_{j d}$ was to ascertain the closeness of forecasts produced via the SDR and MDR analyses. Thus, $M_{d}^{f}$ values for which corresponding $M_{d}^{* f}$ values had very close proximities to those of $z_{j}^{\text {of }}$ were adopted into the set of accepted GFMAPR potential forecasts subject to the accuracy of the method used in fixing the acceptable proximity conditions for screening all possible forecasts.

In this work, a proximity score method was employed in establishing the FAC. Equation (120) infers that a perfect fit between SDR and MDR forecasts will produce zero width of deviation. An initial score was chosen for this forecast scenario. The scoring method for other scenarios was developed from the understanding that $z_{j}^{\text {of }}$ is also a fuzzy value even though its parent state has not been established. $z_{j}^{o f}$ was assumed to exist as the midpoint value of a pseudo-state. The regions bounding the pseudo-state were taken as being equal to the state width value $\omega^{\circ}$. Any $R_{j d}$ value within $-2 \omega^{\circ} \leq R_{j d} \leq 2 \omega^{\circ}$ was awarded a score $e_{j d}$ within the range $0 \leq e_{j d} \leq 10$. Subsequently, it was expected that any $R_{j d}$ existing within $\left(-2 g \omega^{\circ}, 2 g \omega^{\circ}\right)\{g>1\}$ will possess score values in the range $10<e_{j d} \leq \infty$. 
Employing these assumptions and adoptions, a general equation obtained from the interpolation of the identified points and equivalent fixed scores was developed for computing $e_{j d}$ for all $R_{j d}$ :

$e_{j d}=10\left[1-\left(2 \omega^{\circ}\left[1-R_{j d}\right]\right)\right]$

Notice that although $\omega^{\circ}$ was our assumed state width from $z_{j}^{\circ f} 2 \omega^{\circ}$ was employed instead. This was necessary to account for uncertainties and model inadequacies.

For the least deviating forecast to accrue the highest score and the most deviating forecast the least, the outcome from Eq. (121) was employed such that $\min \left(R_{j d}\right)$ was given the score of $\max \left(e_{j d}\right)$ while $\min \left(e_{j d}\right)$ was awarded $\max \left(R_{j d}\right)$. The entire score for $R_{j d}$ was then recomputed.

$$
\begin{aligned}
e_{j d}^{*}= & \min \left(e_{j d}\right)-\left[\left[\left(\max \left(R_{j d}\right)-R_{j d}\right) /\left(\max \left(R_{j d}\right)\right.\right.\right. \\
& \left.\left.\left.-\min \left(R_{j d}\right)\right)\right]\left(\min \left(e_{j d}\right)-\max \left(e_{j d}\right)\right)\right]
\end{aligned}
$$

\section{GFMAPR single value forecast analysis}

The analysis to obtain $x_{n+1}^{f}\left(x_{n+1}^{f}=x^{f}\left(x_{1, n}\right)\right)$ is the final stage of the GFMAPR forecast analysis. Two methods used exclusive of each other for obtaining $x_{n+1}^{f}$ were employed. The first and more prioritised method considered a situation in which the FAC analysis revealed that $\exists R_{j d}:-2 \omega^{\circ} \leq R_{j d} \leq 2 \omega^{\circ}$. The second method applied when the first condition was violated, that is, $\forall R_{j d}:\left(R_{j d}<-2 \omega^{\circ}\right) \oplus\left(R_{j d}>2 \omega^{\circ}\right)$. A procedure which shows how $x_{n+1}^{f}$ was determined using the methods is outlined below.

\section{Procedure for GFMAPR forecast determination}

i. Set initial value of $j(j=1)$

ii. Obtain $R_{j d}\left\{d=1,2,3, \ldots, d^{*}\right\}$.

iii. Apply the FAC criterion. Determine $\max \left(e_{j d}\right)$. Also, obtain corresponding $M_{d}^{f}\left(\max \left(e_{j d}\right)\right)$. Test to see if $\exists R_{j d}:-2 \omega^{\circ} \leq R_{j d} \leq 2 \omega^{\circ}$. If this condition does not exist go to vi.

iv. Compute the proximity score index $\Gamma_{j d}$ for all $e_{j d}$

$\Gamma_{j d}=100 \times\left[\left(\left|\max \left(e_{j d}\right)-e_{j d}\right|\right) / \min \left(\max \left(e_{j d}\right), e_{j d}\right)\right]$

v. If $\Gamma_{j d} \leq 10$, accept $M_{d}^{f}$ into the set of GFMAPR potential forecast $X$.

Let corresponding $e_{j d}$ also be an element in $Y$

vi. If $j<j^{*}$ increase $j$ by a unit value and return to ii. vii. If $j=j^{*}$ then,

$$
\begin{array}{ll}
\text { a. If } & \\
\text { b. } \quad \exists R_{j d}: & : 2 \omega^{\circ} \leq R_{j d} \leq 2 \omega^{\circ}, \text { obtain } x^{f} \text { as, } \\
& x_{n+1}^{f}=\sum_{i=1}^{i^{*}} X_{i}\left(Y_{i} / \sum_{i=1}^{i^{*}} Y_{i}\right)\left\{1 \leq i^{*} \leq j^{*} d^{*}\right\} \\
\forall R_{j d}: & :-2 \omega^{\circ}>R_{j d}>2 \omega^{\circ}, \text { obtain } x^{f} \text { as, } \\
x_{n+1}^{f} & =\sum_{d=1}^{d^{*}} M_{d}^{f}\left(\max \left(e_{j d}^{*}\right) / \sum_{d=1}^{d^{*}} \max \left(e_{j d}^{*}\right)\right)
\end{array}
$$

viii. End procedure

It is worth noting that: $x_{n+1}^{f}=0 \quad\left\{\forall M_{d}^{f}: M_{d}^{f}<0\right\}$

\section{Search procedure for improving GFMAPR forecast}

A preliminary investigation was undertaken to observe the accuracies of GFMAPR forecasts with respect to varying SDR sets. To this end, the set partitioning index $\sigma$ in Eq. (14) was replaced with $\sigma_{i}=\{i=1,2,3, \ldots, \infty\}$, where

$\sigma_{i}=i$

The investigation led to the observation that GFMAPR forecast accuracy had the potential to improve at different $\sigma$ values other than $\sigma=4$. Further experimental investigation of model performances on application to different industrial fire accident data evaluated on the basis of the MAPE showed that better GFMAPR performances could be obtained from the number of created SDR sets related to $\omega_{r}^{*}$

$\omega_{r}^{*}=\omega^{*} / a_{r}$

$a_{r}=r$

$r=\left\{\begin{array}{cl}1 & \left\{\sigma_{i}=\sigma^{*}\right\} \\ a_{r-1}+1 & \left\{\sigma_{i}=\sigma^{*} a_{r-1}\right\}\end{array}\right.$

$\omega^{*}$ is the value of $\omega$ for which one of the set partition indices $\sigma^{*}$, obtained from an initial search using $\sigma_{i}=\{i=$ $1,2,3, \ldots, q\}$ produces the best GFMAPR performance, measured using some performance evaluation approach. $a_{r}$ is a multiplier for $\sigma^{*}$ employed for an extended search for 


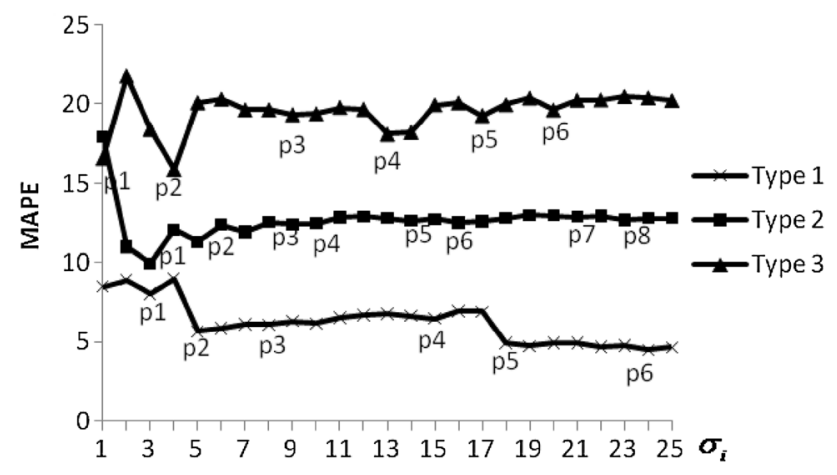

Fig. 4 A plot of GFMAPR (MAPE) performances for a set partition index range of 1-25 using data presented in Table 6

better GFMAPR performances within regions covered by multiples of $\sigma^{*}$.

As an example of how $\sigma^{*}$ was determined in this work, GFMAPR out-of-sample forecasts were carried out at various set partition index values using three industrial accidents occurrences from three literature sources. This was achieved by obtaining the best model MAPE performance at $\sigma_{i}=\{i=1,2,3, \ldots, 5\} . \sigma_{i}$ for which model best forecast was obtained was recorded as $\hat{\sigma}$. Subsequently, the search for improved model performances was widened by further applying the model to obtain forecasts for set partition index values of $v \hat{\sigma} \pm i\{i=1,2, \ldots, \widehat{\sigma}-1\} . v$ is an integer which represents multiples of $\hat{\sigma}\{v \geq 1\} . \sigma^{*}=v \hat{\sigma}$. Figure 4 shows the points of troughs $p$ which indicate model best forecast performances at set partition index regions $\sigma^{*}+i=\left\{1 \leq i<\sigma_{i}\right\}$ (Table 7).

Two observations were made from Fig. 4 . The first is that although the regions where GFMAPR produced relatively high-accurate forecast did not exist as $\sigma^{*}$ multiples, nonetheless a search around the region of $\sigma^{*} a_{r}$ showed potential to improve GFMAPR performances. Second, it was also observed from Type 2 and Type 3 data analysis that the strict use of $\sigma=4$ did not always guarantee the best forecast performance of GFMAPR. The need to improve model performances for all types of industrial accidents data based on these observations justified our interest in developing the GFMAPR search (S-GFMAPR) model. Subsequently, the GFMAPR variant in which $\sigma=4$ is referred to as the GFMAPR non-search (NS-GFMAPR) model.

\section{Development of the comparative performance index for detecting best S-GFMAPR forecasts}

To obtain S-GFMAPR forecasts that are not strictly hinged on a single performance evaluation measure, a comparative performance index (CPI) was developed. The CPI is developed by combining GFMAPR out-of-sample results. This result was determined by the use of a single horizon 
recursive rolling forecast approach (see Sect. 8.2) which was used in computing model MAPE, MAE and MSE result determined during the search process. It was used to distinguish between S-GFMAPR performances computed using two most closely following set partition indices. The superior S-GFMAPR performance was taken as that which produced a lower CPI when any two sets of performances determined within a search region $(r)$ were compared with each other.

For each round of comparison $t_{r}\left\{t_{r}=1,2,3,4, \ldots, t_{r}^{*}\right\}$ the superior performance evaluation index $\operatorname{CPI}^{*}\left(t_{r}\right)$ was obtained as

$$
\begin{aligned}
& \begin{array}{l}
\mathrm{CPI}^{*}(t)=\left\{\begin{array}{l}
\min (\mathrm{CPI}(t-1), \mathrm{CPI}(t)) \\
\min \left(\mathrm{CPI}^{*}(t-1), \mathrm{CPI}(t)\right)
\end{array}\right. \\
\mathrm{CPI}(b)=\left(\operatorname{MAPE}(b) / \sum_{t_{r}-1}^{t_{r}} \operatorname{MAPE}(b)\right)
\end{array} \\
& +\left(\operatorname{MAE}(b) / \sum_{t_{r}-1}^{t_{r}} \operatorname{MAPE}(b)\right) \\
& +\left(\operatorname{MSE}(b) / \sum_{t_{r}-1}^{t_{r}} \operatorname{MAPE}(b)\right)
\end{aligned}
$$

$\operatorname{MAPE}(b)=\left(\sum_{i=5}^{n-4} 100\left|x_{i}-x^{f}\left(x_{(1, i=1)}\right)\right| / x_{i}\right) / n-4$

$\operatorname{MAE}(b)=\left(\sum_{i=5}^{n-4}\left|x_{i}-x^{f}\left(x_{(1, i=1)}\right)\right|\right) / n-4$

$\operatorname{MSE}(b)=\sum_{i=5}^{n-4}\left(x_{i}-x^{f}\left(x_{(1, i=1)}\right)\right)^{2} / n-4$

$\left(b:=t_{r}-1, t_{r}\right)$

$t_{r}$ : is the current period within $r$ region in which GFMAPR results have been evaluated.

$t_{r}-1:$ is the previous period within $r$ in which GFMAPR results have been evaluated.

$N\left(t_{r}\right)$ : is the number of periods/rounds available for search investigation.

$\mathrm{CPI}^{*}\left(t_{r}\right)$ : is the lower CPI value obtained after comparison in the period $t_{r}$.

As a result of the limitation placed on the minimum data size requirement for GFMAPR, the out-of-sample performance index which can be obtained from Eq. (131) can only be computed if an available number of historical data is greater than four. In situations where the historical data number available is equal to the minimum, the S-GFMAPR approach cannot be employed. The NSGFMAPR becomes useful for forecasting in such situations.

\section{Algorithm for undertaking S-GFMAPR forecasting}

Based on conclusions drawn from our preliminary investigation, a procedure for carrying out the search GFMAPR forecasting was developed. The procedure is outlined below using the following steps.

Step 1: Initialise the set partition region search index: $r=0$

Initialise procedure termination signal (PTS) value: PTS $=0$

Step 2: Investigate GFMAPR performances and determine corresponding $\mathrm{CPI}\left(t_{0}\right)$ and $x_{n+1}^{f}\left(t_{0}\right)$ for SDR set partition width $\omega\left(t_{0}\right)$ obtained using initial set partition width regions $\sigma\left(t_{0}\right)\left\{t_{0}=1,2,3, \ldots, t_{0}^{*}\right\}$. In this work, the maximum initial search region value was limited to $t_{0}^{*}=5$

Step 3: Obtain $\sigma^{*}: \sigma^{*}=\sigma\left(\mathrm{CPI}^{*}(r=0)\right) \cdot \mathrm{CPI}^{*}(r=0)$ $=\min \left(\mathrm{CPI}^{*}\left(t_{0}\right)\right)$.

Also, obtain corresponding $x_{n+1}^{f}\left(\mathrm{CPI}^{*}\left(t_{0}\right)\right)$. Then set

$\mathrm{CPI}^{* *}(r)=\mathrm{CPI}^{*}(r)$

$x_{n+1}^{f}\left(\mathrm{CPI}^{* *}(r)\right)=x_{n+1}^{f}\left(\mathrm{CPI}^{*}(r)\right)$

Step 4: Increase $r$ and PTS, respectively, by 1. Obtain $\sigma^{*} a_{r}\left(t_{r}\right),\left(t_{r}=1,2, \ldots, 5\right)$ which represents all search regions within the proximity of $\sigma^{*} a_{r}$ (Table 8 provides the values for $\sigma^{*} a_{r}\left(t_{r}\right)$ ).Carry out GFMAPR forecasts using $\omega\left(\sigma^{*} a_{r}\left(t_{r}\right)\right)$.

Step 5: Determine $\mathrm{CPI}^{*}(r) . \mathrm{CPI}^{*}(r)=\min \left(\mathrm{CPI}\left(t_{r}\right)\right)$. Also, obtain corresponding $x_{n+1}^{f}\left(\mathrm{CPI}^{*} r\right)$

Step 6: Update $\mathrm{CPI}^{* *}(r)$ and obtain corresponding $x_{n+1}^{f}\left(\mathrm{CPI}^{* *}(r)\right)$

$\mathrm{CPI}^{* *}(r)=\min \left(\mathrm{CPI}^{* *}(r-1), \mathrm{CPI}^{*}(r)\right)$

Step 7: If $\mathrm{CPI}^{* *}(r)=\mathrm{CPI}^{*}(r-1)$, increase PTS value by 1. Otherwise, if $\mathrm{CPI}^{* *}(r)=\mathrm{CPI}^{*}(r)$, reset PTS $=0$

Step 8: If PTS $=4$ then GFMAPR forecast $x_{n+1}^{f}$ at the end of the search procedure is

$x_{n+1}^{f}=x_{n+1}^{f}\left(\mathrm{CPI}^{* *}(r)\right)$.

Table 8 Values of set partition indices at multiplier values greater or equal to one

\begin{tabular}{llllll}
\hline$t_{r}$ & 1 & 2 & 3 & 4 & 5 \\
\hline$\sigma^{*} a_{r}\left(t_{r}\right)$ & $\sigma^{*} a_{r}$ & $\sigma^{*} a_{r}+1$ & $\sigma^{*} a_{r}+2$ & $\sigma^{*} a_{r}-1$ & $\sigma^{*} a_{r}-2$ \\
\hline
\end{tabular}


Table 9 Comparison of underground coal mine fatal accidents predictions between GFMAPR performances and the Kher and Yerpude (2015) FTSM Data source: Kher and Yerpude 2015

\begin{tabular}{|c|c|c|c|c|c|c|c|c|c|}
\hline Year & $\begin{array}{l}\text { Number of Fatal } \\
\text { accidents }\end{array}$ & $\begin{array}{l}\text { Kher and } \\
\text { Yerpude }\end{array}$ & $\begin{array}{l}\text { NS- } \\
\text { GFMAPR }\end{array}$ & $\begin{array}{l}\text { S- } \\
\text { GFMAPR }\end{array}$ & Year & $\begin{array}{l}\text { Number of Fatal } \\
\text { accidents }\end{array}$ & $\begin{array}{l}\text { Kher and } \\
\text { Yerpude }\end{array}$ & $\begin{array}{l}\text { NS- } \\
\text { GFMAPR }\end{array}$ & $\begin{array}{l}\text { S- } \\
\text { GFMAPR }\end{array}$ \\
\hline 1990 & 91 & - & - & - & 2002 & 48 & 48 & 48 & 48 \\
\hline 1991 & 80 & - & 80 & 80 & 2003 & 46 & 47 & 46 & 45.5 \\
\hline 1992 & 107 & - & 106 & 107 & 2004 & 49 & 49 & 48 & 49 \\
\hline 1993 & 101 & 100 & 100 & 100.5 & 2005 & 50 & 51 & 50 & 50 \\
\hline 1994 & 93 & 94 & 92 & 93 & 2006 & 44 & 45 & 44 & 44 \\
\hline 1995 & 91 & 91 & 90 & 91 & 2007 & 25 & 26 & 24 & 25 \\
\hline 1996 & 75 & 76 & 74 & 75 & 2008 & 32 & 33 & 32 & 31.5 \\
\hline 1997 & 94 & 95 & 94 & 94 & 2009 & 39 & 40 & 38 & 39 \\
\hline 1998 & 80 & 81 & 80 & 80 & 2010 & 41 & 42 & 40 & 40.5 \\
\hline 1999 & 74 & 75 & 74 & 73.5 & 2011 & 23 & 24 & 24 & 23.5 \\
\hline 2000 & 62 & 62 & 62 & 62 & 2012 & 25 & 26 & 24 & 25 \\
\hline \multirow[t]{2}{*}{2001} & 67 & 68 & 66 & 67 & 2013 & 27 & 28 & 26 & 27 \\
\hline & & & & & 2014 & Forecast & - & 29 & 31 \\
\hline
\end{tabular}

Table 10 GFMAPR in-fit sample performances compared with established models for the underground coal mine fatal accident data

\begin{tabular}{llllllll}
\hline $\begin{array}{l}\text { Model } \\
\text { Method }\end{array}$ & $\begin{array}{l}\text { Grey model } \\
\text { GM }(1,1)\end{array}$ & $\begin{array}{l}\text { ARIMA } \\
(0,1,0)\end{array}$ & $\begin{array}{l}\text { ESM } \\
\text { Holt }\end{array}$ & $\begin{array}{l}\text { Mao and Sun (2011) } \\
\text { Grey-Markov }\end{array}$ & $\begin{array}{l}\text { Kher and Yerpude (2015) } \\
\text { FTSM }\end{array}$ & $\begin{array}{l}\text { NS-GFMAPR } \\
\text { FGM }\end{array}$ & $\begin{array}{l}\text { S-GFMAPR } \\
\text { FGM }\end{array}$ \\
\hline MAE & 9.40 & 8.73 & 7.748 & 1.17 & 0.81 & 0.583 & 0.125 \\
MSE & 88.43 & 0.81 & 0.88 & 3.43 & 0.81 & 0.583 & 0.063 \\
MAPE & 14.14 & 17.87 & 14.98 & 2.87 & 1.87 & 1.30 & 0.30 \\
\hline
\end{tabular}

Table 11 Model results for number of fatalities in yearly traffic accidents from 1974 to 2004 in Belgium Data source: Arutchelvan et al. (2010)

\begin{tabular}{|c|c|c|c|c|c|c|c|c|c|c|c|}
\hline Model & $\begin{array}{l}\text { GM } \\
(1,1)\end{array}$ & ARIMA & ESM & $\begin{array}{l}\text { Lee } \\
\text { et al. } \\
(2007)\end{array}$ & $\begin{array}{l}\text { Jilani and } \\
\text { Bur-ney } \\
(2008)\end{array}$ & $\begin{array}{l}\text { Egrioglu } \\
\text { (2012) }\end{array}$ & $\begin{array}{l}\text { Kamal and } \\
\text { Gihan } \\
(2013)\end{array}$ & $\begin{array}{l}\text { Arutchelvan } \\
\text { et al. (2010) }\end{array}$ & $\begin{array}{l}\text { Mao and } \\
\text { Sun } \\
(2011)\end{array}$ & $\begin{array}{l}\text { NS- } \\
\text { GFMAPR }\end{array}$ & $\begin{array}{l}\text { S- } \\
\text { GFMAPR }\end{array}$ \\
\hline Method & Grey & $(0,1,0)$ & Simple & Fuzzy & Fuzzy & $\begin{array}{l}\text { Fuzzy- } \\
\text { GA }\end{array}$ & $\begin{array}{l}\text { GA/Fuzzy } \\
\text { clustering }\end{array}$ & Fuzzy & $\begin{array}{l}\text { Grey- } \\
\text { Markov }\end{array}$ & GFM & GFM \\
\hline MAE & 95.62 & 76.72 & 75.58 & 72.57 & 70.54 & 28.36 & 16.07 & 7.61 & 8.43 & 6.24 & 2.21 \\
\hline MSE & $9.14 \mathrm{E} 3$ & $7.10 \mathrm{E} 3$ & $7.29 \mathrm{E} 3$ & $6.85 \mathrm{E} 3$ & $6.91 \mathrm{E} 3$ & 978 & 445 & 275.77 & 190.5 & 52.9 & 7.22 \\
\hline MAPE & 5.86 & 5.72 & 5.9 & 5.07 & 5.06 & 2.17 & 1.28 & 0.66 & 0.593 & 0.47 & 0.17 \\
\hline
\end{tabular}

Otherwise, if PTS $<4$ return to step 4 and continue the procedure.

\section{Results and discussion}

The GFMAPR forecasting procedure could be cumbersome and time consuming to undertake manually. In this regard, a Visual Basic.Net program was created to execute the multiple procedures contained in the model.

Model performances were investigated on two fronts, namely accuracy of the developed set creation and partitioning method which was measured using the insample (trained model) prediction evaluation, and the forecast capability of the model which was investigated using the out-of-sample forecast method.

\section{GFMAPR data set partitioning accuracy in model training}

In-sample fitted results obtained using the GFMAPR (SDR) set partitioning technique was compared using two industrial accident data (Zheng and Liu 2009; Kher and Yerpude 2015) and one traffic accident data (Arutchelvan et al. 2010) The traffic accident data were adopted on the 
Table 12 GFMAPR data in-fit results compared with those of established methods using a chemical plant accident occurrence data Data source: Zheng and Liu (2009)

\begin{tabular}{lcccccccc}
\hline Model & ARIMA & Regression & ESM & Grey & ANN & Grey-Markov & NS-GFMAPR & S-GFMAPR \\
\hline MAE & 40.25 & 35.22 & 30.67 & 29.48 & 14.57 & 6.0 & 1.25 & 0.50 \\
MSE & $3.42 \mathrm{E} 3$ & $2.39 \mathrm{E} 3$ & $1.79 \mathrm{E} 3$ & $1.42 \mathrm{E} 3$ & $4.67 \mathrm{E} 2$ & 69.69 & 4.38 & 0.25 \\
MAPE & 24.19 & 16.96 & 19.57 & 18.67 & 10.07 & 3.83 & 1.59 & 0.417 \\
\hline
\end{tabular}

basis that traffic accident occurrences also exhibit random and uncertain patterns. In addition, the traffic accident data have been employed in the analysis of several set partitioning models (Lee et al. 2007; Jilani and Burney 2008; Egrioglu 2012; Kamal and Gihan 2013). Table 9 shows actual in-sample (trained model) predictions for one of the accident occurrence data that were analysed, while Tables 10, 11 and 12 show the NS-GFMAPR and S-GFMAPR prediction fitness to data used in building the model in comparison with results obtained by the established models previously mentioned.

It can be observed from the presented results that the set partitioning technique developed in the GFMAPR model produces the best fit to data when compared to various results obtained from commonly employed models employed for accidents prediction.

\section{Forecast capability of GFMAPR}

It can be observed from Table 9 that the apart from predicting trained data outputs, the developed model can also be employed for making future predictions (referred to in this paper as forecasts). However, the model is limited to making forecasts for single horizon (one step ahead) only. That is, given an available dataset of $n\{n \geq 4\}$ occurrences, GFMAPR can only forecast accident occurrences for the period $n+1$ and not beyond that. Thus, the model may not be suitable for making multiple horizons forecasting or model out-of sample tests except in situations where bootstrapping methods are utilised. As a fallout of this limitation, splitting a dataset into model training and testing portions become unnecessary. Thus, for a given dataset of $n$ occurrences, $n$ number of data points are also required for training GFMAPR. This property of the model imposes a restriction on proper model validation with respect to the out-of-sample prediction or forecast capability of GFMAPR. In overcoming these shortcomings, the out-ofsample forecast evaluation method was adopted for model evaluation and validation.

The out-of-sample forecast evaluation exploits the single forecast horizon capability of GFMAPR using a recursive rolling mechanism. This means that for any data sample of size $n$ with occurrences in horizon $k[x(k)\{k=1,2,3, \ldots, n\}]$, the forecast $x^{f}(k)$ was obtained for a trained split data set $x_{(1, k)}(k=4)$ and tested against $x_{(1, k)}$. The split data set was subsequently increased by a single data point and the training and single horizon forecast (testing) process repeated for $k=5,6,7, \ldots$, $k=n-1$.

The PE indices $\left(\mathrm{PE}_{m i j}\right)$ for GFMAPR $(m)$ in terms of the absolute error (AE), absolute percentage error (APE) and square error (SE) were determined using $x^{f}(i)$ and $x_{i+1}\{i=k, k+1, k+2, \ldots, n-1: k \geq 4\} . \quad \mathrm{PE}_{m i j}^{o o s} \quad$ for each model variant was then obtained as,

$\mathrm{PE}_{m i j}^{o o s}=\sum_{i=k}^{n-1} \mathrm{PE}_{m i j} /(n-4) \quad\{j \in J\}$

\section{$J:\{\mathrm{AE}, \mathrm{APE}, \mathrm{SE}\}$}

The capability of the GFMAPR variants for single horizon forecasting was subsequently investigated by comparing their forecast performances with results produced by six forecasting models with forecasting capability commonly used or designed for use in industrial accidents forecasting. The established models employed for the analysis were namely: ARIMA, ESM, a three-point M.A model, GM $(1,1)$, Grey-Markov model (Mao and Sun 2011) and DPEWTA (Edem et al. 2016).

The model validation and evaluation involve a comparison of the results of the GFMAPR model variants with those of the mentioned models on their application to some industrial accidents data using the out-of-sample forecasting approach previously discussed.

In carrying the evaluation, a PE weight index $\varpi_{m}$ was created by converting the MAPE, MSE and MAE values obtained for all the models into relative weight values $C_{m j}$ (Eq. 141). The sum of these relative weights was then deployed as the PE index for the model variants as well as the compared models.

$$
\begin{gathered}
\varpi_{m}=\sum_{j=1}^{N(J)} C_{m j} \quad\{m=1,2,3, \ldots, N(M)\} \\
C_{m j}=\left(\mathrm{PE}_{m i j}^{o o s} / \sum_{j=1}^{N(M)} \mathrm{PE}_{m i j}^{o o s}\right)^{-1} \\
\{m=1,2,3, \ldots, N(M) ; j=1,2, \ldots, N(J)\}
\end{gathered}
$$


Table 13 Developed and compared model forecasts of the underground coal mine fatal accidents

\begin{tabular}{|c|c|c|c|c|c|c|c|c|c|}
\hline \multirow[t]{2}{*}{ Year } & \multirow[t]{2}{*}{ Number of accidents } & \multicolumn{6}{|c|}{ Compared models } & \multicolumn{2}{|c|}{ Developed model variants } \\
\hline & & ARIMA & ESM & MA & DPEWTA & Grey & Grey-Markov & NS-GFMAPR & S-GFMAPR \\
\hline 1990 & 91 & - & - & - & - & - & - & - & - \\
\hline 1991 & 80 & - & - & - & - & - & - & - & - \\
\hline 1992 & 107 & - & - & - & - & - & - & - & - \\
\hline 1993 & 101 & - & - & - & - & - & - & - & - \\
\hline 1994 & 93 & 94 & 97 & 96 & 100 & 118 & 124 & 94 & 94 \\
\hline 1995 & 91 & 95 & 93 & 95 & 97 & 103 & 106 & 101 & 85 \\
\hline 1996 & 75 & 94 & 92 & 98 & 90 & 97 & 96 & 89 & 88 \\
\hline 1997 & 94 & 91 & 89 & 86 & 67 & 84 & 86 & 75 & 74 \\
\hline 1998 & 80 & 91 & 93 & 92 & 84 & 87 & 88 & 98 & 97 \\
\hline 1999 & 74 & 91 & 89 & 92 & 88 & 83 & 84 & 79 & 77 \\
\hline 2000 & 62 & 87 & 86 & 83 & 76 & 78 & 77 & 77 & 76 \\
\hline 2001 & 67 & 76 & 70 & 72 & 58 & 71 & 66 & 68 & 67 \\
\hline 2002 & 48 & 75 & 67 & 68 & 68 & 67 & 66 & 64 & 64 \\
\hline 2003 & 46 & 44 & 57 & 59 & 59 & 60 & 48 & 48 & 47 \\
\hline 2004 & 49 & 43 & 38 & 47 & 48 & 55 & 50 & 48 & 47 \\
\hline 2005 & 50 & 46 & 49 & 48 & 50 & 51 & 47 & 54 & 56 \\
\hline 2006 & 44 & 47 & 44 & 50 & 50 & 48 & 49 & 52 & 46 \\
\hline 2007 & 25 & 41 & 40 & 48 & 48 & 45 & 42 & 45 & 41 \\
\hline 2008 & 32 & 21 & 33 & 35 & 37 & 40 & 29 & 22 & 23 \\
\hline 2009 & 39 & 29 & 29 & 29 & 34 & 37 & 31 & 33 & 29 \\
\hline 2010 & 41 & 36 & 27 & 32 & 36 & 35 & 36 & 42 & 42 \\
\hline 2011 & 23 & 39 & 26 & 40 & 41 & 34 & 38 & 46 & 40 \\
\hline 2012 & 25 & 22 & 23 & 34 & 33 & 31 & 22 & 25 & 24 \\
\hline 2013 & 27 & 24 & 19 & 24 & 26 & 29 & 24 & 31 & 26 \\
\hline 2014 & Future forecast & 27 & 17 & 26 & 27 & 27 & 26 & 29 & 31 \\
\hline
\end{tabular}

Table 14 Forecasting performance for the underground coal mine fatal accidents data

\begin{tabular}{llllcccrr}
\hline Model & ARIMA & ESM & MA & DPEWTA & Grey & Grey-Markov & NS-GFMAPR & S-GFMAPR \\
\hline MAE & 9.770 & 8.298 & 10.550 & 10.050 & 10.200 & 9.600 & 8.900 & 7.800 \\
MSE $(\times$ E2) & 1.556 & 1.259 & 1.632 & 1.564 & 1.515 & 1.537 & 1.328 & 1.065 \\
MAPE & 21.993 & 18.918 & 24.455 & 22.749 & 21.504 & 19.760 & 20.784 & 17.606 \\
$\varpi_{m}$ & 22.684 & 27.023 & 21.007 & 22.181 & 22.732 & 23.774 & 25.144 & 3 \\
Rank & 6 & 2 & 8 & 7 & 5 & 4 & 0.0469 & 1 \\
CT (s) & - & - & 0.0020 & 0.0156 & 0.0156 & 0.3438 & 0.322 \\
\hline
\end{tabular}

$N(M)$ is the number of models employed for comparative analysis

$N(J)$ is the number of performance evaluation methods employed for comparative analysis

This approach was necessary as a result of the need to utilise a single PE index which exhibited the combined characteristics of the MAPE, MSE and MAE in the evaluation of the models.
Using the out-of-sample forecast evaluation approach, the forecasts made by the developed and compared models when applied on the three accidents historical data sets previously employed in Sect. 8.1 were obtained. The out-of sample forecasts for the three industrial accidents data employed in this work are as shown in Tables 13, 15 and 17. The forecast performance evaluation results obtained from the three accidents historical data sets using this technique are shown in Tables 14, 16 and 18. 
Table 15 Developed and compared model forecasts for the number of fatalities in yearly traffic accidents from 1974 to 2004 in Belgium

\begin{tabular}{|c|c|c|c|c|c|c|c|c|c|}
\hline \multirow[t]{2}{*}{ Year } & \multirow[t]{2}{*}{ Number of accidents } & \multicolumn{6}{|c|}{ Compared models } & \multicolumn{2}{|c|}{ Developed model variants } \\
\hline & & ARIMA & ESM & MA & DPEWTA & Grey & Grey-Markov & NS-GFMAPR & S-GFMAPR \\
\hline 1974 & 1574 & - & - & - & - & - & - & - & - \\
\hline 1975 & 1460 & - & - & - & - & - & - & - & - \\
\hline 1976 & 1536 & - & - & - & - & - & - & - & - \\
\hline 1977 & 1597 & - & - & - & - & - & - & - & - \\
\hline 1978 & 1644 & 1510 & 1553 & 1567 & 1569 & 1673 & 1681 & 1656 & 1677 \\
\hline 1979 & 1572 & 1593 & 1579 & 1621 & 1660 & 1718 & 1727 & 1686 & 1707 \\
\hline 1980 & 1616 & 1566 & 1572 & 1562 & 1544 & 1662 & 1637 & 1622 & 1634 \\
\hline 1981 & 1564 & 1584 & 1581 & 1593 & 1598 & 1665 & 1673 & 1647 & 1585 \\
\hline 1982 & 1464 & 1569 & 1564 & 1570 & 1538 & 1633 & 1625 & 1616 & 1519 \\
\hline 1983 & 1479 & 1536 & 1552 & 1514 & 1522 & 1565 & 1549 & 1519 & 1516 \\
\hline 1984 & 1369 & 1522 & 1541 & 1472 & 1497 & 1529 & 1514 & 1498 & 1555 \\
\hline 1985 & 1308 & 1349 & 1389 & 1424 & 1419 & 1464 & 1435 & 1400 & 1424 \\
\hline 1986 & 1456 & 1284 & 1263 & 1339 & 1334 & 1401 & 1338 & 1325 & 1388 \\
\hline 1987 & 1390 & 1446 & 1424 & 1382 & 1546 & 1400 & 1414 & 1454 & 1441 \\
\hline 1988 & 1432 & 1376 & 1399 & 1423 & 1412 & 1382 & 1396 & 1384 & 1393 \\
\hline 1989 & 1488 & 1422 & 1422 & 1411 & 1449 & 1380 & 1387 & 1412 & 1426 \\
\hline 1990 & 1574 & 1482 & 1470 & 1460 & 1465 & 1392 & 1441 & 1486 & 1518 \\
\hline 1991 & 1471 & 1574 & 1555 & 1531 & 1622 & 1423 & 1501 & 1562 & 1512 \\
\hline 1992 & 1380 & 1484 & 1492 & 1523 & 1530 & 1426 & 1419 & 1463 & 1470 \\
\hline 1993 & 1346 & 1424 & 1406 & 1426 & 1431 & 1408 & 1429 & 1428 & 1346 \\
\hline 1994 & 1415 & 1334 & 1353 & 1363 & 1339 & 1388 & 1353 & 1418 & 1404 \\
\hline 1995 & 1228 & 1407 & 1403 & 1381 & 1455 & 1384 & 1377 & 1415 & 1420 \\
\hline 1996 & 1122 & 1212 & 1274 & 1322 & 1337 & 1348 & 1233 & 1207 & 1203 \\
\hline 1997 & 1150 & 1101 & 1130 & 1175 & 1196 & 1301 & 1158 & 1213 & 1152 \\
\hline 1998 & 1224 & 1132 & 1148 & 1136 & 1151 & 1266 & 1152 & 1164 & 1150 \\
\hline 1999 & 1173 & 1209 & 1217 & 1187 & 1185 & 1247 & 1228 & 1216 & 1247 \\
\hline 2000 & 1253 & 1157 & 1178 & 1199 & 1197 & 1223 & 1174 & 1183 & 1182 \\
\hline 2001 & 1288 & 1241 & 1243 & 1213 & 1298 & 1214 & 1232 & 1252 & 1254 \\
\hline 2002 & 1145 & 1277 & 1283 & 1271 & 1279 & 1210 & 1252 & 1349 & 1341 \\
\hline 2003 & 1035 & 1130 & 1168 & 1217 & 1248 & 1189 & 1147 & 1147 & 1123 \\
\hline 2004 & 953 & 1016 & 1038 & 1090 & 1093 & 1159 & 1026 & 1096 & 1076 \\
\hline 2005 & & 932 & 953 & 994 & 916 & 1123 & 983 & 974 & 1005 \\
\hline
\end{tabular}

Table 16 Forecasting performance for the number of fatalities in yearly traffic accidents from 1974 to 2004 in Belgium

\begin{tabular}{|c|c|c|c|c|c|c|c|c|}
\hline Model & ARIMA & ESM & MA & DPEWTA & Grey & Grey-Markov & NS-GFMAPR & S-GFMAPR \\
\hline MAE & 84.000 & 84.296 & 84.556 & 98.482 & 98.482 & 84.185 & 85.074 & 72.37 \\
\hline MSE $(\times$ E4) & 8.798 & 9.525 & 9.734 & 13.28 & 13.407 & 9.038 & 9.677 & 8.149 \\
\hline MAPE & 6.347 & 6.475 & 6.617 & 7.66 & 7.677 & 6.34 & 6.601 & 5.661 \\
\hline$\varpi_{m}$ & 25.917 & 25.014 & 45.057 & 20.135 & 20.061 & 25.662 & 24.647 & 28.998 \\
\hline Rank & 4 & 3 & 6 & 7 & 8 & 2 & 5 & 1 \\
\hline CT (s) & - & - & 0.0019 & 0.0156 & 0.0156 & 0.5156 & 0.0313 & 1.4495 \\
\hline
\end{tabular}

It was observed from the results presented in Tables 14, 16 and 18 that in terms of the $\varpi_{m}$ which combines the accuracies of the models MAE, MSE and MAPE into a single evaluation score (Eq. 135); S-GFMAPR produced the best out-of-sample forecast results in all but one of the data set analysed. In terms of the performance score, 
Table 17 Developed and compared model forecasts of the a chemical plant accident occurrence data

\begin{tabular}{|c|c|c|c|c|c|c|c|c|c|}
\hline \multirow[t]{2}{*}{ Year } & \multirow[t]{2}{*}{ Number of accidents } & \multicolumn{6}{|c|}{ Compared models } & \multicolumn{2}{|c|}{ Developed model variants } \\
\hline & & ARIMA & ESM & MA & DPEWTA & Grey & Grey-Markov & NS-GFMAPR & S-GFMAPR \\
\hline 2000 & 350 & - & - & - & - & - & - & - & - \\
\hline 2001 & 347 & - & - & - & - & - & - & - & - \\
\hline 2002 & 437 & - & - & - & - & - & - & - & - \\
\hline 2003 & 260 & - & - & - & - & - & - & - & - \\
\hline 2004 & 211 & 272 & 335 & 349 & 272 & 278 & 261 & 282 & 260 \\
\hline 2005 & 215 & 270 & 299 & 236 & 238 & 202 & 192 & 227 & 211 \\
\hline 2006 & 214 & 259 & 220 & 213 & 240 & 177 & 167 & 234 & 238 \\
\hline 2007 & 191 & 251 & 215 & 215 & 226 & 165 & 163 & 236 & 234 \\
\hline 2008 & 109 & 241 & 197 & 203 & 202 & 152 & 158 & 205 & 201 \\
\hline 2009 & 109 & 218 & 124 & 150 & 52 & 122 & 102 & 111 & 117 \\
\hline 2010 & 112 & 223 & 111 & 109 & 94 & 103 & 102 & 123 & 106 \\
\hline 2011 & 63 & 214 & 58 & 111 & 118 & 90 & 89 & 125 & 130 \\
\hline 2012 & 57 & 202 & 31 & 88 & 111 & 74 & 58 & 75 & 53 \\
\hline 2013 & 40 & 192 & 11 & 60 & 61 & 62 & 49 & 61 & 55 \\
\hline 2014 & Forecast & 48 & 0 & 49 & 34 & 51 & 37 & 42 & 40 \\
\hline
\end{tabular}

Table 18 Forecasting performance for the chemical plant accident occurrence data

\begin{tabular}{lllllllll}
\hline Model & ARIMA & ESM & MA & DPEWTA & Grey & Grey-Markov & NS-GFMAPR & S-GFMAPR \\
\hline MAE & 62.100 & 40.310 & 42.100 & 44.300 & 27.4 & 25 & 35.800 & 31.200 \\
MSE (× E3) & 9.158 & 3.262 & 3.425 & 2.476 & 1.03 & 0.933 & 2.156 & 1.814 \\
MAPE & 41.969 & 33.605 & 39.531 & 45.831 & 25.5796 & 19.6846 & 35.436 & 30.679 \\
$\varpi_{m}$ & 14.100 & 23.189 & 21.290 & 22.696 & 55.564 & 64.173 & 27.543 & 32.128 \\
Rank & 8 & 5 & 7 & 6 & 2 & 1 & 3 & 3 \\
CT (s) & - & - & 0.0000 & 0.0000 & 0.0000 & 0.3125 & 0.0156 & 0.2969 \\
\hline
\end{tabular}

S-GFMAPR produced the highest score in two of the three analysed data with a minimum and maximum positive relative score difference of about ten percent and seventy percent, respectively.

The model, however, produced inferior results when compared with grey and Grey-Markov models on application to the chemical plant accidents data.

The general results obtained indicate that although S-GFMAPR may not be suitable for forecasting all forms of industrial accident occurrences, it does exhibit superiority over other compared models when applied on data with characteristics suitable for S-GFMAPR forecasting.

However, the computation time (CT) for GFMAPR models using a $2.13 \mathrm{GHz}$ Intel Pentium P6200 CPU with $4 \mathrm{~GB}$ of RAM is generally higher compared to all other models. Nonetheless, the CT range of 1 and $2.5 \mathrm{~s}$ can be considered as acceptable tradeoffs for the models excellent forecasting capabilities. As expected, NS-GFMAPR produced results less superior to those of S-GFMAPR. However, when compared in terms of all eight analysed models, its general performance score point ranking fell between 3 and 4. This implies that although it is less effective than S-GFMAPR, it can still be employed for forecasting. NSGFMAPR will be more suitable in situations where time efficient forecasting is a necessity, and when available historical data points are limited to four. To further ensure the validity of the GFMAPR variants, the models were applied on five more real life data related to industrial and traffic accident obtained from literature sources ("Appendix G"). The results obtained further strengthen the validity of NS-GFMAPR and S-GFMAPR as 
suitable models for forecasting fluctuating, random and uncertain occurrences.

\section{Investigation to ascertain GFMAPR forecast capability given varying historical data sizes and shocks}

Although preliminary investigation showed that the GFMAPR model possessed huge potential for more accurate small data sample size forecasting, a more exhaustive investigation was undertaken to ascertain this observation. Consideration was given to small (4-15), medium (16-35) and large $(>35)$ sample size of data. Due to the unavailability of industrial accident occurrence records of very large sizes, simulated data were employed.

Thirty data sets, each made up of seventy periods was simulated. To ensure that the simulated data possessed real industrial accident occurrence characteristics, properties of historical industrial accidents data obtained from the literature were investigated and employed for the simulation. The properties investigated for include the degree of shocks and the frequency of fluctuation in occurrences. Each data set characteristic was simulated using the information turbulence index $(\theta)$ and the Quad point characteristic trace QPCT approach (Edem et al. 2016) such that each possessed some degree of shocks within the range of $0<\theta \leq 0.4$ and fluctuation index $\left(\eta^{F}\right)$ ranging between $0<\eta^{F} \leq 1$ (Eq. 89). The recursive rolling single horizon out-of-sample forecasts were undertaken for S-GFMAPR, ARIMA, ESM, MA, Grey-Markov and DPEWTA models.

GFMAPR forecast MAPE performance relative to each of the models employed for comparative analysis $\left(R_{m d k}\right)$ was then computed as

$$
\begin{aligned}
& \left(R_{m d k}\right)=100 \times\left(\left[\left(\mathrm{PE}_{d k j}^{o o s}+1\right)-\left(\mathrm{PE}_{m d k j}^{o o s}+1\right)\right] /\left(\mathrm{PE}_{d k j}^{o o s}+1\right)\right) \\
& (d=1,2,3, \ldots, 30 ; k=4,5,6, \ldots, 70 ; m \in M)
\end{aligned}
$$

where $M:\{$ All models used for comparative analysis with GFMAPR $\}, j:$ MAPE

The constant unit value in Eq. (142) was added to the relation to counter situations of zero MAPE values. The mean GFMAPR-compared model performance was subsequently obtained as,

$\bar{R}_{m d k}=\left(\sum_{d=1}^{30} R_{m d k}\right) / 30 \quad\{k=4,5,6, \ldots, 70\}$

A graphical plot of $\bar{R}_{m d k}$ against $k$ was then undertaken for each $m$.

At the end of the analysis, it was observed that GFMAPR exhibited forecast superiority over all compared

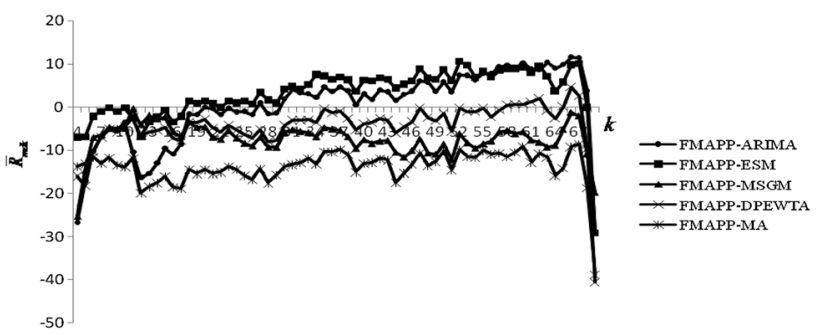

Fig. 5 GFMAPR relative MAPE performances with respect to increasing data size

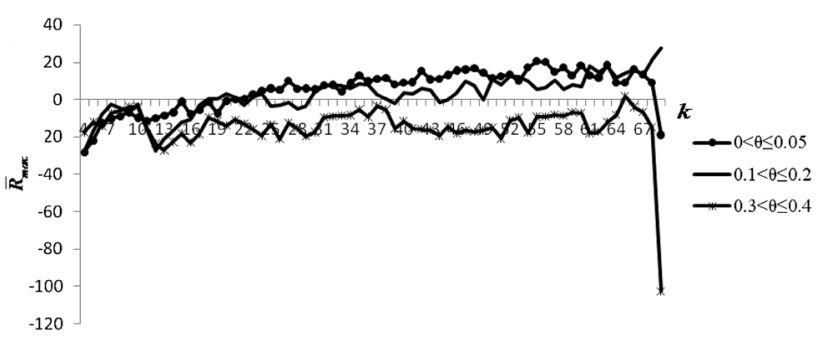

Fig. 6 GFMAPR-ARIMA relative MAPE performances for different degree of variation in data in the presence of increasing data size

models with respect to small data size forecasting. It also showed superior performance over grey, grey-Markov and DPEWTA models with respect to medium and large data sizes. However, ARIMA and ESM exhibited superior forecast performance over GFMAPR within data size range between 28 and 70 (Fig. 5). Note that negative values of $\bar{R}_{m d k}$ imply superior GFMAPR performance.

Furthermore, the simulated data sets were separated into five equal groups based on their degree of variation characteristics. Their MAPE performances were also observed as they changed with data size. The MAPE for GFMAPR relative to each compared model showed that GFMAPR forecasts increasingly improved with increasing degree of variation in data. For example, Fig. 6 shows the relative GFMAPR-ARIMA forecasts for increasing $\theta$ values. It can be easily seen from the figures that at values of $\theta>0.3$, GFMAPR models become more reliable for undertaking forecasts. A summary of the results obtained from this and analysis is presented in Table 21.

The mean $(\bar{F})$ and standard deviations $\left(\sigma_{F}\right)$ of the GFMAPR forecast accuracies on its application to the simulated data samples given different variations and size of data samples are presented in Table 20. It is believed that these results are statistically valid since thirty datasets were simulated for each data point that makes up a sample size class. The obtained results further strengthen the capability of the model for accident forecasting.

It should be noted here that the all forecast results obtained are acceptable within a single forecast horizon which is the focus of this work. 
Table 19 Mean percentage relative superior and non-superior performance of GFMAPR to compared models for varying degree of data variations and different data sizes

\begin{tabular}{|c|c|c|c|c|c|c|}
\hline Data size & $0 \leq \theta \leq 0.5$ & $0.05 \leq \theta \leq 0.1-3.61$ & & $0.1 \leq \theta \leq 0.2$ & $0.2 \leq \theta \leq 0.3$ & $0.3 \leq \theta \leq 0.4$ \\
\hline \multirow[t]{5}{*}{ Small $4 \leq k \leq 15$} & ARIMA & -11.39 & -9.54 & -13.52 & -9.68 & -14.34 \\
\hline & ESM & -3.61 & -2.48 & -1.73 & 0.04 & -6.68 \\
\hline & MA & -23.82 & -21.94 & -13.77 & -9.16 & -4.16 \\
\hline & MSGM & -20.21 & -15.13 & -12.23 & -5.20 & -15.25 \\
\hline & DPEWTA & -4.63 & -6.87 & -13.52 & -1.25 & -11.81 \\
\hline \multirow[t]{5}{*}{ Medium $16 \leq k \leq 35$} & ARIMA & 3.26 & 2.17 & 0.92 & 4.33 & -13.83 \\
\hline & ESM & 6.06 & 2.12 & 2.24 & 7.65 & -7.77 \\
\hline & MA & -22.34 & -21.31 & -13.97 & -6.13 & -10.51 \\
\hline & MSGM & -18.84 & -27.48 & -22.38 & -6.34 & -19.34 \\
\hline & DPEWTA & 3.16 & -4.28 & -7.63 & 2.17 & -17.41 \\
\hline \multirow[t]{5}{*}{ Large $36 \leq k \leq 70$} & ARIMA & 12.38 & 6.29 & 8.81 & 13.24 & -14.75 \\
\hline & ESM & 14.93 & 8.15 & 4.89 & 17.80 & -16.06 \\
\hline & MA & -17.36 & -21.15 & -11.54 & 1.35 & -16.23 \\
\hline & MSGM & -7.88 & -41.25 & -118.21 & -73.33 & -33.66 \\
\hline & DPEWTA & 7.91 & 4.10 & -4.56 & 4.21 & -25.82 \\
\hline
\end{tabular}

Negative and positive values indicate relative superiority and inferiority, respectively, of developed model to compared model

Table 20 The mean and standard deviation GFMAPR forecast accuracies in relation accident occurrence variation and sample sizes

\begin{tabular}{llllrrr}
\hline Data size & Forecast & $0 \leq \theta \leq 0.5$ & $0.05 \leq \theta \leq 0.1$ & $0.1 \leq \theta \leq 0.2$ & $0.2 \leq \theta \leq 0.3$ & $0.3 \leq \theta \leq 0.4$ \\
\hline Small $4 \leq k \leq 15)$ & Mean & 2.191 & 5.624 & 14.514 & 27.836 & 38.544 \\
& Standard deviation & 2.199 & 2.401 & 4.801 & 9.676 & 15.035 \\
Medium $16 \leq k \leq 35$ & Mean & 2.748 & 7.712 & 15.788 & 25.773 & 34.882 \\
& Standard deviation & 2.865 & 0.714 & 4.074 & 3.871 & 6.410 \\
Large 36 $\leq k \leq 70$ & Mean & 3.425 & 7.854 & 15.987 & 27.355 & 41.556 \\
& Standard deviation & 3.459 & 0.807 & 2.998 & 2.684 & 8.298 \\
\hline
\end{tabular}

\section{Application of the developed model on real-time industrial accident occurrences}

The validation of the models with respect to their capability to accurately forecast industrial accidents was also investigated. This was achieved by the application of the model to a case of real-time industrial accidents occurrence data.

The historical information for the case study represents the accidents data of a cocoa processing firm operating in the south-west region of Nigeria. The data obtained covered operating periods within 2003-2015. The required data for the model were collected from the vetting of accident records of various departments operating in the organisation. During the process of data collection, the accidents were observed to occur from sources such as actions and inactions related to poor housekeeping, lack of proper communication during equipment repair and maintenance and delay in the elimination of identified hazards.
GFMAPR forecasts results obtained from the analysis of the case study are presented in Table 19. Based on Lewis's subjective MAPE interpretations (Ofori et al. 2012), the model variants produce MAPE results that indicate reasonable forecasting. In addition, considering the data size (14 periods) and the degree variation in occurrences $(\theta=0.2592)$ the GFMAPR forecasts evaluated in terms of the MAPE can be considered satisfactory since it was found to lay within an expected range of $\bar{F} \pm$ $1 \sigma_{F}(4 \leq k \leq 15 ; 0.2 \leq \theta \leq 0.3$ ) (See Table 20).

The MAPE result may be deceptive due to its scale sensitivity in the presence of a low volume of accident occurrences which is the case of the organisation under study. In line with Stellwagen (2011) recommendation on the tests of model validity in such situations, the model forecast evaluated using the MAE shows forecast accuracy that lies within the accident occurrence range of \pm 3 and \pm 2 for NS-GFMAPR and S-GFMAPR, respectively. This 
Table 21 Results obtained on application of GFMAPR variants on cocoa processing firm accident occurrences

\begin{tabular}{lllll}
\hline Year & Historical occurrences & Trained model predictions & \multicolumn{2}{l}{ Out-of-sample forecast (rolling mechanism) } \\
\cline { 4 - 5 } & & & NS-GFMAPR & S-GFMAPR \\
\hline 2004 & 12 & 12 & - & - \\
2005 & 9 & 9 & - & - \\
2006 & 11 & 11 & - & - \\
2007 & 16 & 16 & - & - \\
2008 & 9 & 9 & 13 & 13 \\
2009 & 10 & 10 & 11 & 11 \\
2010 & 12 & 12 & 11 & 12 \\
2011 & 14 & 14 & 12 & 11 \\
2012 & 8 & 8 & 14 & 13 \\
2013 & 6 & 6 & 11 & 9 \\
2014 & 6 & 6 & 8 & 7 \\
2015 & 7 & 7 & 8 & 8 \\
2016 & Future forecast & - & 8 & 7 \\
& MAPE & 0.000 & 35.377 & 27.416 \\
& MSE & 0.000 & 11.000 & 7.750 \\
& MAE & 0.000 & 2.750 & 2.250 \\
\hline
\end{tabular}

result further strengthens the accuracy of the model (Table 21).

\section{Advantages and disadvantages of GFMAPR}

The GFMAPR model proposed in the current paper has a number of advantages put side-by-side of earlier developed models. Here, the GFMAPR is noted as an excellent model in the absence of exhaustive data. In fact, it generally exhibits superior short and medium data length forecasting quality over all models employed for comparative analysis. This is substantial as a merit, and one of the strongest attributes of the model. The GFMAPR does not require many technicalities in execution. Interestingly, GFMAPR requires only the input information. Data pattern analysis and detection are executed by the model. Another merit of the model is that it does not require parametric input to describe data characteristics. It is also noteworthy that GFMAPR model exhibits improved forecasting ability overall compared models when applied on data characterised by strong variations.

Regrettably, even with the enormous advantages of using the GFMAPR model over other competing ones, it has a number of weaknesses. First, the model is incapable of undertaking out-of-sample forecasting for periods longer than one forecast horizon. Second, computational experience during the analysis of the model results showed that it is difficult to execute manually without the aid of a computer device. A third drawback of the model is that it is less efficient in terms of computation time when compared with other established models analysed in this work. It was also observed during the model analysis that GFMAPR model was less effective when employed for large size data forecasting. This is a disadvantage. Nevertheless, this disadvantage may be ignored since the primary aim of developing the model was hinged on the need to make accurate forecasts in the absence of extensive data sizes.

\section{Conclusions and future works}

A fuzzy set classification based method which employs grey, Markov and pattern recognition concepts for forecasting industrial accident occurrences has been developed. The in-fit-sample and out-of-sample forecast performance of the model was investigated in comparison with some forecasting models frequently employed in the industry by their application on three real-time industrial accidents related data. In addition, the forecast capability of the model with regards to data sizes, variation and fluctuating characteristics were also investigated with the use of simulated industrial accident occurrence scenarios.

In this communication, our purpose is to present an understanding of the first analysis from both the theoretical perspective and a practical application viewpoint of the conceived theory of grey-fuzzy-Markov pattern recognition model for accident forecasting under reasonable assumptions. This investigation broadens the horizon on accident forecasting in industrial settings by showing that accident data could be read into patterns and successfully decomposed and then using the transitional Markov 
property to detect the possible vibrational direction of future accidents. The GFMAPR model was developed to enquire about the linked characteristics of grey, fuzzy, Markov and pattern recognition.

Through several case examinations against published literature a real-time data and, the main conclusions are as follows:

- GFMAPR shows excellent forecast capability for data characterised by fluctuations, variations and randomness;

- GFMAPR is quite effective for forecasting in the presence of small and medium sized data availability;

- GFMAPR is able to make relatively high accurate forecasts when compared against models frequently employment in the industry for forecasting; and

- GFMAPR is thus suitable and reliable for industrial accident prediction.

This paper has made important contributions to research as well as practice. On the first note, an original method based on grey-fuzzy-Markov pattern recognition model was developed to track uncertainties, imprecision and randomness in historical data. Secondly, a new summative data analysis, which involves data preparation, the creation of summative variation data relationship (SDR), fuzzification and reclassifications of data based on the degree of variation was developed. A practical step in evaluating the association among data elements was established for the first time. Similarly, the multiplicative data relationship (MDR) analysis was established as a first-time contribution in accident forecasting literature. This investigation, in search for a procedure in enhancing GFMAPR forecast, developed a comparative performance index for detecting the best S-GFMAPR forecasts for the first time in literature. Furthermore, it is the first report in literature, on accident forecasting, to have an algorithm for understanding the novel S-GFMAPR forecasting, and this has been clearly detailed out in the current paper. Now, considering the contributions of this paper, it hoped that this report will serve as a reference document, as procedural paper that guides further investigations in further probing the details of the foundations and in so doing; we will have a rich number of papers that may develop into an area in industrial accident forecast. Consequently, this study has a practical importance for industrial systems to work out and execute an accident forecasting model that accounts for uncertainties, imprecision and randomness in their systems. Based on this, industrial organisations would acquire the will of monitoring and planning fully for any accidents that would occur in their systems.
Researching to unveil the potential of grey-fuzzyMarkov models with respect to accident forecasting is definitely a new topic in the safety arena. There are lots of opportunities for future research on the topic. Future investigations could be directed at improving the industrial accidents forecast by studying how combined or hybrid models can be developed using GFMAPR for the purpose of producing more improved forecast performance. In addition, further research could be made towards the use of GFMAPR for multiple horizon forecasting. It may also be worthwhile in finding out the performance attribute of realtime data collected from rarely studied systems on accidents such as maintenance functions as much as accidents appear to be due to poor maintenance actions in organisations probably due to inefficiency, poorly trained skilled workers some other causes.

In this communication, theoretical results have been provided based on the attributes of the GMFAPR model using the combined characteristics of grey, fuzzy, Markov and pattern recognition. Forecast performance of the GFMAPR model was compared model for the varying degrees of data variation range as $0 \leq \theta \leq 0.5,0.05 \leq \theta \leq 0.1,0.1 \leq \theta \leq 0.2,0.2 \leq \theta \leq 0.3$ and $0.3 \leq \theta \leq 0.4$ while the data size range according to small $4 \leq k \leq 15$ medium $16 \leq k \leq 35$ and large $36 \leq k \leq 70$. It was shown that forecast results using GFMAPR were progressively enhanced with small and medium size data and with elevations in the degree of fluctuation and variation attributes for the explored data. The theoretical model outcomes were demonstrated through scrutinising the performance of models as they were related in comparison with GFMAPR using models as ARIMA, ESM, MA, MSGM and DPEWTA. Thus, from the quantitative analysis using data sets, the forecast with support from GFMAPR are reasonable with the aforementioned alternatives presently in vogue.

Open Access This article is distributed under the terms of the Creative Commons Attribution 4.0 International License (http://crea tivecommons.org/licenses/by/4.0/), which permits unrestricted use, distribution, and reproduction in any medium, provided you give appropriate credit to the original author(s) and the source, provide a link to the Creative Commons license, and indicate if changes were made.

\section{Appendix A}

Let $\bar{D}\left(\delta_{i, i+1}\right) \quad$ exist as row vector $\bar{D}_{j}\{j=i: i=1,2,3, \ldots, n-1\}$. Also, let the point for which $\bar{D}_{j}=0$ be $m$. In addition, set the initial smoothing cycle counter $v=0$ such that the vector within any procedure cycle is expressed as $\bar{D}_{j}(v)$. The smoothing procedure can be carried out as follows: 
1. Increase smoothing cycle counter by 1 . Carry out a top to bottom inspection of $\bar{D}_{j}(v-1)$

a. if point $m$ exists, then

i. If $m=1$ or $m=n-1$

$$
\begin{aligned}
& \bar{D}_{m}(v)=\left\{\begin{array}{c}
1 \\
-1
\end{array}\left\{\begin{array}{l}
\left.\rho^{+}\left(\bar{D}_{g}(v-1)\right)\right\} \\
\left.\rho^{-}\left(\bar{D}_{g}(v-1)\right)\right\}
\end{array}\right\}\right. \\
& \bar{D}_{g}(v)\left\{\begin{array}{l}
\bar{D}_{g}(v-1)-1\left\{\rho^{+}\left(\bar{D}_{g}(v-1)\right) ;\left|\bar{D}_{g}(v-1)\right| \geq 1\right\} \\
\bar{D}_{g}(v-1)+1\left\{\rho^{-}\left(\bar{D}_{g}(v-1)\right) ;\left|\bar{D}_{g}(v-1)\right| \geq 1\right\}
\end{array}\right.
\end{aligned}
$$

$$
\begin{aligned}
& \left\{g=m+1, m+2, \ldots, g^{*} ;\{m=1\}, \rho\left(\bar{D}_{g *}(v-1)\right) \leftrightarrow \rho\left(\bar{D}_{m}(v)\right)\right\} \\
& \left\{g=m-1, m-2, \ldots, g^{*} ;\{m=n-1\}, \rho\left(\bar{D}_{g^{*}}(v-1)\right) \leftrightarrow \rho\left(\bar{D}_{m}(v)\right)\right\} \\
& \left|\bar{D}_{g^{*}}(v-1)\right| \geq 1
\end{aligned}
$$

Return to 1

ii. If $m \neq 1$ and $m \neq n-1$ then If $\exists \bar{D}_{j}(v-1)$ : $\left|\bar{D}_{j}(v-1)\right|>1$ Obtain $\bar{D}_{q}(v-1)$ and

$\bar{D}_{w}(v-1)$

$\bar{D}_{q}(v-1)=\bar{D}_{g}(v-1)$

$$
\left\{\left|\bar{D}_{g}(v-1)>1, g=m+1, m+2, \ldots, g^{*}\right|\right\}
$$

$$
\begin{aligned}
& \bar{D}_{w}(v-1)=\bar{D}_{g}(v-1) \\
& \quad\left\{\left|\bar{D}_{g}(v-1)>1, g=m+1, m-2, \ldots, g^{*}\right|\right\}
\end{aligned}
$$

Then,

$$
\begin{aligned}
& \bar{D}_{g}(v)=\left\{\begin{array}{l}
1 \\
-1 \\
\bar{D}_{g}(v-1)-1 \\
\bar{D}_{g}(v-1)+1
\end{array} \quad\left\{\begin{array}{l}
\left.\rho^{+}\left(\bar{D}_{g}(v-1)\right),(g \neq q) \oplus(g \neq w)\right\} \\
\left.\rho^{-}\left(\bar{D}_{g}(v-1)\right),(g \neq q) \oplus(g \neq w)\right\} \\
\left.\rho^{-}\left(\bar{D}_{g}(v)\right),(g=q) \oplus(g=w)\right\}
\end{array}\right\}\right. \\
& \left\{g=m+1, m+2, \ldots, g^{*} ;\left|\bar{D}_{q}(v-1)\right|>\left|\bar{D}_{w}(v-1)\right|\right\}
\end{aligned}
$$

Return to 1

iii. If $m \neq 1$ and $m \neq n-1$, then if $\max$ $\left(\left|\bar{D}_{j}(v-1)\right|\right)=1$ then equation (A1) is employed in adjusting $\quad \bar{D}_{m}(v) g=m+1, m+2, \ldots, g^{*} ; \bar{D}_{g^{*}} \neq 0$ $\overline{\mathrm{D}}_{\mathrm{m}}(v)$. Return to 1 .

b. If $m$ does not exist, end procedure.

It is worth noting from the smoothing procedure that one or more of these static variation scenarios can exist in $\bar{D}_{j}$ given single or multiple non-variation points. The approach here is to employ the procedure in eliminating each nonvarying point. Thus, for $T$ non-varying points to be eliminated, then $T$ cycles of the smoothing procedure will be applied. $g^{*}$ is the termination point of the procedure for one cycle.

\section{Appendix B}

Relations for obtaining current, maximum, and minimum variation pattern swing impulses

i. Current pattern swing impulse:

$r_{\mathrm{cur}}^{I}= \begin{cases}\sum_{j=n}^{\tau} F\left(r_{j}^{L}\right) & \left\{F\left(r_{n}^{L}\right)=1 ; \rho\left(r_{n}^{L}\right) \leftrightarrow \rho\left(r_{f}^{L}\right)\right\} \\ 0 & \{\text { otherwise }\}\end{cases}$

$\tau=j \quad\left\{\left(r_{j-1}^{L}=0\right) \oplus\left[\rho\left(r_{j-1}^{L}\right) \uparrow \rho\left(r_{f}^{L}\right)\right]\right\}$

$F\left(r_{j}^{L}\right)=\left\{\begin{array}{ll}1 & \left\{r_{j}^{L} \neq 0\right\} \\ 0 & \text { \{otherwise }\end{array} \quad r:=E, U\right.$.

ii. Maximum, and minimum pattern swing impulses for escalation and closure-lag:

$r_{\min }^{I}=\min \left(\hat{r}_{i}\right) \cdot r_{\max }^{I}=\max \left(\hat{r}_{i}\right)$,

$\begin{aligned} \hat{r}_{i} & =\left\{\begin{array}{l}\sum_{j=m(i)}^{m *(i)} F\left(r_{j}^{L}\right) \cdot m(i) \\ 0 \quad \text { otherwise }\}\end{array}\right. \\ & =j\left\{r_{j}^{L} \neq 0 ; \rho\left(r_{j}^{L}\right) \leftrightarrow \rho\left(r_{f}^{L}\right)\right\} .\end{aligned}$

$m^{*}(i)=j+1 \quad\left\{\left(r_{j+1}^{L}=0\right) \oplus\left(\rho\left(r_{j+1}^{L}\right) \uparrow \rho\left(r_{f}^{L}\right)\right)\right\}$. $m_{i}^{*}>m_{i}$.

$F\left(r_{j}^{L}\right)=\left\{\begin{array}{ll}1 & \left\{r_{j}^{L} \neq 0\right. \\ 0 & \text { \{otherwise }\}\end{array}\right.$. $j=1,2,3, \ldots, n-1 r:=E, U$. 
iii. Maximum, and minimum pattern swing impulses for exact-closure:

$$
\begin{aligned}
& C_{\min }^{I}=\min \left(\hat{C}_{i}\right) \cdot C_{\max }^{I}=\max \left(\hat{C}_{i}\right) \\
& \hat{C}_{i}=p(i)-q(i) \cdot p(i)=j\left\{F\left(O_{j}^{L}\right)=1\right\} \cdot q(i) \\
& \quad=j\left\{F\left(C_{j}^{L}\right)=1\right\} . \\
& q(i)>p(i) \cdot p(i+1)=q(i)+1\{i>1\} . \\
& F\left(r_{j}^{L}\right)=\left\{\begin{array}{c}
1\left\{r_{j}^{L} \neq 0\right\} \\
0\{\text { otherwise }\}
\end{array} . j=1,2,3, \ldots, n-1\right.
\end{aligned}
$$

\section{Appendix C}

Adjustment relations for escalation and closure-lag patterns to account for overlap properties

$$
E_{j}^{* L}=\left\{\begin{array}{l}
E_{j}^{L}\left\{F\left(E_{j}^{L}\right)=1 ; \rho\left(E_{j}^{L}\right) \leftrightarrow \rho\left(E_{f}^{L}\right)\right\} \\
O_{j}^{* L}\left\{F\left(E_{j}^{L}\right)=0 ; F\left(O_{j}^{L}\right)=1 ;\left|O_{j}^{* L}\right|<\left|L^{E V}\right| ; \rho\left(O_{j}^{L}\right) \leftrightarrow \rho\left(E_{f}^{L}\right)\right\} \\
V_{j}^{* L}\left\{F\left(E_{j}^{L}\right)=0 ; F\left(V_{j}^{L}\right)=1 ;\left|V_{j}^{* L}\right|<\left|L^{E V}\right| ; \rho\left(V_{j}^{L}\right) \leftrightarrow \rho\left(E_{f}^{L}\right)\right\} \\
\mathrm{L}^{E V}\{P\} \mid\{Q\}
\end{array}\right.
$$

$$
U_{j}^{* L}=\left\{\begin{array}{l}
U_{j}^{L}\left\{F\left(U_{j}^{L}\right)=1 ; \rho\left(U_{j}^{L}\right) \leftrightarrow \rho\left(U_{f}^{L}\right)\right\} \\
C_{j}^{* L}\left\{F\left(U_{j}^{L}\right)=0 ; F\left(C_{j}^{L}\right)=1 ;\left|C_{j}^{* L}\right|<\left|L^{U}\right| ; \rho\left(C_{j}^{L}\right) \leftrightarrow \rho\left(U_{f}^{L}\right)\right\} \\
\mathrm{L}^{E V}\left\{F\left(U_{j}^{L}\right)=0 ; F\left(C_{j}^{L}\right)=1 ;\left|C_{j}^{* L}\right| \geq\left|L^{U}\right| ; \rho\left(C_{j}^{L}\right) \leftrightarrow \rho\left(U_{f}^{L}\right)\right\}
\end{array}\right.
$$

where,

$\bar{r}^{L}=\left|\left(\sum_{j=1}^{n} j \times r_{j}^{L} / \sum_{j=1}^{n} j\right)\right|\left\{F\left(r_{j}^{L}\right)=1, \rho\left(r_{j}^{L}\right) \leftrightarrow \rho\left(r_{f}^{L}\right)\right\}, r:=O, C, V$

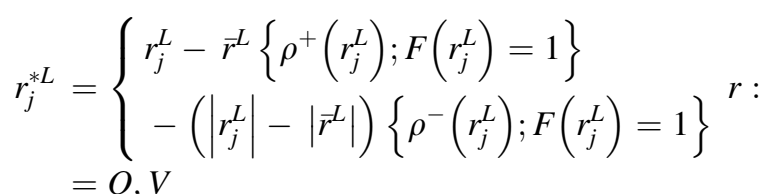

$$
C_{j}^{* L}=\left\{\begin{array}{l}
\bar{C}^{L}-1\left\{\rho^{+}\left(C_{j}^{L}\right) ; F\left(C_{j}^{L}\right)=1 ; C_{j}^{L}>\bar{C}^{L}\right\} \\
1-\left|\bar{C}^{L}\right|\left\{\rho^{-}\left(C_{j}^{L}\right) ; F\left(C_{j}^{L}\right)=1 ;\left|C_{j}^{L}\right|>\left|\bar{C}^{L}\right|\right\} \\
C_{j}^{L}\{T\} \mid\{W\}
\end{array}\right.
$$

$$
\begin{aligned}
P & \equiv F\left(E_{j}^{L}\right)=0 ; F\left(O_{j}^{L}\right)=1 ;\left|O_{j}^{* L}\right| \geq\left|L^{E V}\right| ; \rho\left(O_{j}^{L}\right) \\
& \leftrightarrow \rho\left(E_{f}^{L}\right)
\end{aligned}
$$

Table 22 Values of $r_{\text {cur }}^{\theta L}$ adopted for various $r_{\text {cur }}^{\theta L U B}$ and $r_{\text {cur }}^{\theta L L B}$ conditions given expected pattern future swing direction

\begin{tabular}{lll}
\hline Adopted $r_{\text {cur }}^{\theta L}(r \equiv E, U)$ & $r_{\text {cur }}^{\theta L}$ boundary conditions & $b^{*}$ condition \\
\hline$r_{\text {cur }}^{\theta L U B}$ & $h \leq r_{\text {cur }}^{\theta L U B}, r_{\text {cur }}^{\theta L L B} \leq H ; r_{\text {cur }}^{\theta L U B} \leq r_{\text {cur }}^{\theta L L B} ; \rho^{+}\left(r_{f}^{L}\right)$ & $b^{*}>1$ \\
& $h \leq r_{\text {cur }}^{\theta L U B} \leq H ;\left(r_{\text {cur }}^{\theta L L B}<h\right) \oplus\left(r_{\text {cur }}^{\theta L L B}>H\right) ; \rho^{+}\left(r_{f}^{L}\right)$ \\
& $h \leq r_{\text {cur }}^{\theta L U B}, r_{\text {cur }}^{\theta L L B} \leq H ; r_{\text {cur }}^{\theta L U B} \leq r_{\text {cur }}^{\theta L L B} ; \rho^{-}\left(r_{f}^{L}\right)$ \\
$-r_{\text {cur }}^{\theta L U B}$ & $h \leq r_{\text {cur }}^{\theta L U B} \leq H ;\left(r_{\text {cur }}^{\theta L L B}<h\right) \oplus\left(r_{\text {cur }}^{\theta L L B}>H\right) ; \rho^{-}\left(r_{f}^{L}\right)$ \\
& $h \leq r_{\text {cur }}^{\theta L U B}, r_{\text {cur }}^{\theta L L B} \leq H ; r_{\text {cur }}^{\theta L U B}>r_{\text {cur }}^{\theta L L B} ; \rho^{+}\left(r_{f}^{L}\right)$ \\
$r_{\text {cur }}^{\theta L L B}$ & $h \leq r_{\text {cur }}^{\theta L L B} \leq H ;\left(r_{\text {cur }}^{\theta L U B}<h\right) \oplus\left(r_{\text {cur }}^{\theta L U B}>H\right) ; \rho^{+}\left(r_{f}^{L}\right)$ \\
& $h \leq r_{\text {cur }}^{\theta L U B}, r_{\text {cur }}^{\theta L L B} \leq H ; r_{\text {cur }}^{\theta L U B}>r_{\text {cur }}^{\theta L L B} ; \rho^{-}\left(r_{f}^{L}\right)$ \\
$-r_{\text {cur }}^{\theta L L B}$ & $h \leq r_{\text {cur }}^{\theta L L B} \leq H ;\left(r_{\text {cur }}^{\theta L U B}<h\right) \oplus\left(r_{\text {cur }}^{\theta L U B}>H\right) ; \rho^{-}\left(r_{f}^{L}\right)$ \\
& $\left(r_{\text {cur }}^{\theta L U B}, r_{\text {cur }}^{\theta L L B}<h\right) \oplus\left(r_{\text {cur }}^{\theta L U B}, r_{\text {cur }}^{\theta L L B}>H\right) ; \rho^{+}\left(r_{f}^{L}\right)$ \\
$r_{b^{*}}^{\psi L L}$ & $\left(r_{\text {cur }}^{\theta L U B}, r_{\text {cur }}^{\theta L L B}<h\right) \oplus\left(r_{\text {cur }}^{\theta L U B}, r_{\text {cur }}^{\theta L L B}>H\right) ; \rho^{-}\left(r_{f}^{L}\right)$ \\
$-r_{b^{*}}^{\psi L L}$ & $\rho^{+}\left(r_{f}^{L}\right) \rho^{-}\left(r_{f}^{L}\right)$ \\
0 &
\end{tabular}




$$
\begin{aligned}
& \begin{array}{l}
Q \equiv F\left(E_{j}^{L}\right)=0 ; F\left(V_{j}^{L}\right)=1 ;\left|V_{j}^{* L}\right| \geq\left|L^{E V}\right| ; \rho\left(V_{j}^{L}\right) \\
\leftrightarrow \rho\left(E_{f}^{L}\right)
\end{array} \\
& T \equiv \rho^{+}\left(C_{j}^{L}\right) ; F\left(C_{j}^{L}\right)=1 ; C_{j}^{L} \leq \bar{C}^{L} . \\
& W \equiv \rho^{-}\left(C_{j}^{L}\right) ; F\left(C_{j}^{L}\right)=1 ;\left|C_{j}^{L}\right| \leq\left|\bar{C}^{L}\right| \\
& \mathrm{L}^{E V}=\left\{\begin{array}{l}
\max \left(\max \left(E_{j}^{L}\right), \max \left(V_{j}^{L}\right)\right)\left\{\rho^{+}\left(E_{f}^{L}\right)\right\} \\
\min \left(\min \left(E_{j}^{L}\right), \min \left(V_{j}^{L}\right)\right)\{\text { otherwise }\}
\end{array}\right. \\
& \mathrm{L}^{U}=\left\{\begin{array}{l}
\max \left(U_{j}^{L}\right)\left\{\rho^{+}\left(U_{f}^{L}\right)\right\} \\
\min \left(U_{j}^{L}\right)\{\text { otherwise }\}
\end{array}\right.
\end{aligned}
$$

\section{Appendix D}

Procedure for the estimation of current and equivalent cumulative swing magnitudes for escalating and closinglag patterns (see Table 22)

i. Determination of $E_{\text {cur }}^{* L}$ and $U_{\text {cur }}^{* L}$

$$
\begin{aligned}
& r_{\text {cur }}^{* L}=\left\{\begin{array}{l}
\sum_{j=n}^{\tau} r_{j}^{* L}\left\{F\left(r_{n}^{* L}\right)=1 ; \rho\left(r_{n}^{* L}\right) \leftrightarrow \rho\left(r_{f}^{L}\right)\right\} \\
0\{\text { otherwise }\}
\end{array}\right. \\
& \tau=j\left\{\left(r_{j-1}^{* L}=0\right) \oplus\left[\rho\left(r_{j-1}^{* L}\right) \uparrow \rho\left(r_{f}^{L}\right)\right]\right\}, r:=E, U
\end{aligned}
$$

ii. Determination of $E_{\text {cur }}^{\theta L}$ and $U_{\text {cur }}^{\theta L}$ :

The following steps are employed in estimating $E_{\text {cur }}^{\theta L}$ and $U_{\text {cur }}^{\theta L}$ is outlined below using the following steps,

Step 1: Obtain $r_{b}^{\prime}$ from $r_{j}^{* L}$

$r_{b}^{\prime}=r_{j}^{* L}\left\{F\left(r_{j}^{* L}\right)=1\right\}$

Step 2: Using time weights, adjust absolute $r_{b}^{\prime}$ to become $r_{b}^{\prime \prime}$

$r_{b}^{\prime \prime}=b \times\left|r_{b}^{\prime}\right|$

Step 3: Obtain $\mathrm{H}$ and $\mathrm{h}$, respectively,

$$
\begin{gathered}
H=\max \left(r_{b}^{\prime \prime}\right) \\
h=\min \left(r_{b}^{\prime \psi}\right)
\end{gathered}
$$

Step 4: Obtain $r_{b}^{\prime \psi}$ variation index:

$\vec{r}_{b}^{\psi}=\sum_{b=1}^{b^{*}-1}\left(r_{b}^{\prime \psi}-r_{b+1}^{\prime \psi}\right) /\left(b^{*}-1\right)$

$r_{\text {cur }}^{\theta L}$ will be a value existing between $\left(r_{\text {cur }}^{\theta L U B}\right.$ and $\left.r_{\text {cur }}^{\theta L L B}\right)$ :

$r_{\mathrm{cur}}^{\theta L U B}=\left(r_{b^{*}}^{\prime \psi}+\vec{r}^{\prime \psi}\right) / b^{*}$.

$r_{\text {cur }}^{\theta L L B}=\left(r_{b^{*}}^{\psi}-\vec{r}^{\prime \psi}\right) / b^{*}$

$r:=E, U . b=1, \ldots, b^{*} .1 \leq b^{*} \leq n$

\section{Appendix E}

Rules and relations employed in obtaining $r_{f}^{L}$ value using the PSM technique

S/N Method name $\quad \begin{aligned} & \text { Conditions guiding choice by } \\ & \text { method }\end{aligned}$

1 Non-static forecast consideration

$$
r_{f}^{L}=\left\{\begin{array}{c}
A\left\{A \neq r_{n}^{\Phi}, B=r_{n}^{\Phi}\right\} \\
B\left\{B \neq r_{n}^{\Phi}, A=r_{n}^{\Phi}\right\}
\end{array}\right.
$$

2 Minimum proximity to swing span $[T P(g)]$

$$
r_{f}^{L}=\left\{\begin{array}{c}
A\{T P(A)<T P(B)\} \\
B\{T P(B)<T P(A)\}
\end{array}\right.
$$

3 Minimum $g$ deviation $\begin{aligned} & \text { Minimum } g \text { deviation } \\ & \text { from } r_{j}^{\Phi} \text { span based on } \\ & \text { time-weighted average }\end{aligned} \quad r_{f}^{L}=\left\{\begin{array}{l}A\{|A-\bar{P}(t)|<|B-\bar{P}(t)|\} \\ B\{|B-\bar{P}(t)|<|A-\bar{P}(t)|\}\end{array}\right.$ method $[\bar{P}(t)]$ $4 \quad \begin{gathered}\text { Minimum } g \text { deviation } \\ \text { from } r_{j}^{\Phi} \text { span based on }\end{gathered} r_{f}^{L}=\left\{\begin{array}{l}A\{|A-\bar{P}|<|B-\bar{P}|\} \\ B\{|B-\bar{P}|<|A-\bar{P}|\}\end{array}\right.$ weighted average method $(\bar{P})$

$5 \quad$ Use of $r_{n}^{\Phi}$ as future $\quad r_{f}^{L}=r_{n}^{\Phi}$ \{all other explored pattern value conditions have been violated $\}$

$$
\begin{aligned}
& T P(g)=\sum\left|r_{j}^{\Phi}-r_{j+1}^{\Phi}\right| \cdot \bar{P}=\sum_{j=1}^{n-2} j \times\left(\left|r_{j}^{\Phi}-r_{j+1}^{\Phi}\right|\right) / \sum_{j=1}^{n-2} j \\
& \bar{P}=\sum_{j=1}^{n-2}\left(\left|r_{j}^{\Phi}-r_{j+1}^{\Phi}\right|\right) /(n-2) \cdot g=A, B
\end{aligned}
$$

Note that the elimination approaches are arranged in their order of preference of use. If a more preferred method is explored and found to be suitable for use, then the other methods are subsequently ignored. Otherwise the next preferred method is explored.

\section{Appendix F}

Future variation pattern verification relations

1. Future escalation pattern: 


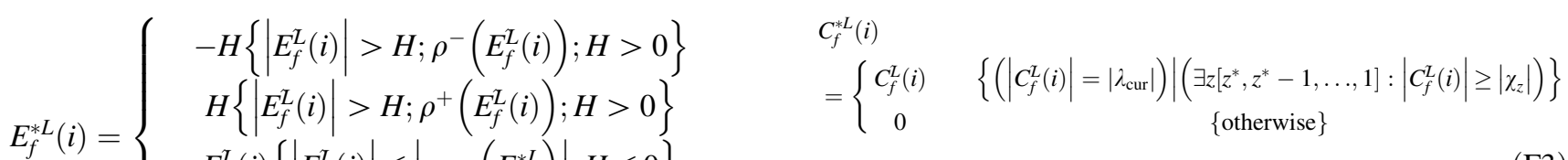

$$
\begin{aligned}
& E_{f}^{L}(i)\left\{\left|E_{f}^{\mathcal{L}}(i)\right| \leq\left|\max \left(E_{j}^{* L}\right)\right| ; H \leq 0\right\} \\
& \max \left(E_{j}^{* L}\right)\left\{\left|E_{f}^{L}(i)\right|>\left|\max \left(E_{j}^{* L}\right)\right| ; H \leq 0\right\} \quad \text { 4. Future closing-lead } \\
& H=\left\{\begin{array}{cl}
\max \left(E_{j}^{* L}\right)-\left|\lambda_{\text {cur }}\right| & \left\{\rho^{+}\left(E_{f}^{L}\right)\right\} \\
\left|\min \left(E_{j}^{* L}\right)\right|-\left|\lambda_{\text {cur }}\right| & \text { \{otherwise }\}
\end{array}\right. \\
& V_{f}^{* L}(i)=\left(V_{f a}^{* L}(i)\right) \mid\left(V_{f b}^{* L}(i)\left\{V_{f a}^{* L}(i)=0\right\}\right), \\
& V_{f a}^{* L}(i)=\left\{\begin{array}{l}
D\{A ; B ; C ; D \leq M\} \mid\{A ; F ; L ; D<N\} \\
0\{\text { otherwise }\}
\end{array}\right.
\end{aligned}
$$

2. Future closure-lag pattern:

$U_{f}^{* L}(i)=\left\{\begin{aligned} U_{f}^{L}(i) & \left\{\left|U_{f}^{L}(i)\right|<\left|\lambda_{\text {cur }}\right|\right\} \\ 0 & \text { \{therwise }\}\end{aligned}\right.$

$$
V_{f b}^{* L}(i)=\left\{\begin{array}{l}
D^{\prime}\left(\min \left[\left|D^{\prime}\right|-|W|\right]\right)\left\{A^{\prime} ; B ; C ; D^{\prime}<M\right\} \oplus\left\{A^{\prime} ; F ; L ; D^{\prime}<N\right\} \\
0\{\text { otherwise }\}
\end{array}\right.
$$

\begin{tabular}{|c|c|c|c|c|c|c|c|c|}
\hline Model & ARIMA & ESM & MA & DPEWTA & Grey & Grey-Markov & NS-GFMAPR & S-GFMAPR \\
\hline MAE $(\times \mathrm{E} 4)$ & 2.212 & 1.874 & 2.728 & 1.988 & 2.513 & 2.304 & 2.291 & 1.515 \\
\hline MSE (× E8) & 8.597 & 6.263 & 11.05 & 4.233 & 9.438 & 8.021 & 6.929. & 3.391 \\
\hline MAPE & 9.32 & 7.75 & 11.15 & 8.16 & 9.93 & 9.23 & 8.90 & 5.89 \\
\hline$\varpi_{m}$ & 22.15 & 27.61 & 17.96 & 31.08 & 20.14 & 22.39 & 23.85 & 40.52 \\
\hline Rank & 6 & 3 & 8 & 2 & 7 & 5 & 4 & 1 \\
\hline CT (s) & 0.0020 & 0.0020 & 0.0341 & 0.0624 & 0.0000 & 0.0780 & 0.9652 & 1.5001 \\
\hline
\end{tabular}

3. Future exact-closure pattern

$$
W=\sum_{j=1}^{n} j V_{j}^{L} / \sum_{j=1}^{n} j\left\{F\left(V_{j}^{L}\right)=1 ; \rho\left(V_{j}^{L}\right) \leftrightarrow \rho\left(E_{f}^{L}\right)\right\}
$$

Table 23 Future forecasting performance for data on fire accidents occurring in PR China between 1997 and 2004 Data source: Jiang (2007)

\begin{tabular}{|c|c|c|c|c|c|c|c|c|}
\hline Model & ARIMA & ESM & MA & DPEWTA & Grey & Grey-Markov & NS-GFMAPR & S-GFMAPR \\
\hline MAE & 15.89 & 16.32 & 18.00 & 16.91 & 24.18 & 22.55 & 11.18 & 11.18 \\
\hline MSE & 341.25 & 336.27 & 501.27 & 565.27 & 902.91 & 872.73 & 320.64 & 320.64 \\
\hline MAPE & 16.39 & 17.17 & 15.89 & 15.19 & 21.01 & 20.35 & 9.30 & 9.30 \\
\hline$\varpi_{m}$ & 28.37 & 27.98 & 23.71 & 23.62 & 16.17 & 16.93 & 38.56 & 38.56 \\
\hline Rank & 3 & 4 & 5 & 6 & 8 & 7 & 1 & 1 \\
\hline CT (s) & - & - & 0.0037 & 0.0626 & 0.0000 & 0.8594 & 1.0000 & 1.2500 \\
\hline
\end{tabular}

Table 24 Future forecasting performance for data on yearly accident injuries in Ghana for the period 1991-2011 Data Source: Ofori et al. (2012)

\begin{tabular}{llllccccc}
\hline Model & ARIMA & ESM & MA & DPEWTA & Grey & Grey-Markov & NS-GFMAPR & S-GFMAPR \\
\hline MAE & $2.64 \mathrm{E} 3$ & $2.53 \mathrm{E} 3$ & $2.08 \mathrm{E} 3$ & $2.28 \mathrm{E} 3$ & $2.51 \mathrm{E} 3$ & $2.57 \mathrm{E} 3$ & $2.19 \mathrm{E} 3$ & $1.97 \mathrm{E} 3$ \\
MSE & $9.38 \mathrm{E} 6$ & $8.57 \mathrm{E} 6$ & $8.85 \mathrm{E} 6$ & $7.12 \mathrm{E} 6$ & $9.45 \mathrm{E} 6$ & $8.56 \mathrm{E} 6$ & $2.67 \mathrm{E} 6$ & $5.95 \mathrm{E} 6$ \\
MAPE & 27.28 & 26.71 & 20.46 & 22.53 & 28.54 & 28.25 & 21.48 & 19.54 \\
$\varpi_{m}$ & 21.40 & 22.33 & 25.92 & 26.05 & 21.21 & 21.83 & 6.15 & 30.48 \\
Rank & 7 & 5 & 4 & 3 & 8 & 0.7032 & 0.9935 & 1 \\
CT $(\mathrm{s})$ & - & - & 0.0731 & 0.0938 & 0.0000 & 0.4374 \\
\hline
\end{tabular}

Table 25 Future forecasting performance for Data on fire accident occurrences in the oil and gas industry for the period 1996-2010 Data Source: Docstoc (2013) 
Table 26 Future forecasting performance for data on construction industry safety production indicator gas industry for the period Jan. 2010 to Dec. 2011 Data Source: Yabo et al. (2012)

\begin{tabular}{llllccccc}
\hline Model & ARIMA & ESM & MA & DPEWTA & Grey & Grey-Markov & NS-GFMAPR & S-GFMAPR \\
\hline MAE & 0.075 & 0.070 & 0.094 & 0.079 & 0.096 & 0.086 & 0.085 & 0.077 \\
MSE & 0.007 & 0.006 & 0.012 & 0.010 & 0.013 & 0.013 & 0.012 & 10.89 \\
MAPE & 9.61 & 8.73 & 12.06 & 10.12 & 11.78 & 10.89 & 22.41 & 5.80 \\
$\varpi_{m}$ & 29.41 & 32.90 & 20.91 & 24.97 & 20.40 & 21.78 & 6 & 25.46 \\
Rank & 2 & 1 & 5 & 4 & 7 & 0.0000 & 0.9375 & 1.0157 \\
CT (s) & - & - & 0.0021 & 0.0781 & & 1.6407 \\
\hline
\end{tabular}

Table 27 Future forecasting performance for data on total number of fire accidents in China for the period 2001-2011 Data Source: Huang et al. (2012a, b)

\begin{tabular}{|c|c|c|c|c|c|c|c|c|}
\hline Model & ARIMA & ESM & MA & DPEWTA & Grey & Grey-Markov & NS-GFMAPR & S-GFMAPR \\
\hline MAE & 36.67 & 26.58 & 28.00 & 25.14 & 41.57 & 39.29 & 24.57 & 24.43 \\
\hline MSE & $2.52 \mathrm{E} 3$ & $1.67 \mathrm{E} 3$ & $1.57 \mathrm{E} 3$ & $1.32 \mathrm{E} 3$ & $2.54 \mathrm{E} 3$ & $2.36 \mathrm{E} 3$ & $1.50 \mathrm{E} 3$ & $1.49 \mathrm{E} 3$ \\
\hline MAPE & 34.85 & 22.30 & 26.56 & 24.95 & 47.62 & 44.74 & 23.19 & 22.41 \\
\hline$\varpi_{m}$ & 19.73 & 29.29 & 27.62 & 31.02 & 17.00 & 18.12 & 30.64 & 31.13 \\
\hline Rank & 6 & 4 & 5 & 2 & 8 & 7 & 3 & 1 \\
\hline $\mathrm{CT}$ & - & - & 0.0012 & 0.0625 & 0.0000 & 0.2031 & 1.0122 & 1.1719 \\
\hline
\end{tabular}

$$
\begin{aligned}
A & \equiv\left|V_{f}^{* L}(i)\right|>\left|\lambda_{\text {cur }}\right|, \quad B \equiv \rho^{-}\left(V_{f}^{* L}(i)\right) \\
& C \equiv \min \left(V_{j}^{L}\right) \neq 0, \quad D \equiv V_{f}^{* L}(i)+\lambda_{\text {cur }}, \\
M & =\left|\min \left(V_{j}^{L}\right)\right|+1, F \equiv \rho^{+}\left(V_{f}^{* L}(i)\right), L \equiv \max \left(V_{j}^{L}\right) / \\
& =0, N \equiv \max \left(V_{j}^{L}\right)+1
\end{aligned}
$$

$A^{\prime} \equiv\left|V_{f}^{* L}(i)\right|>\left|\chi_{z}\right|, \quad D^{\prime} \equiv\left|V_{f}^{* L}(i)\right|+\left|\chi_{z}\right|$

$i=1,2, \ldots, i^{*}, \quad \mathrm{z}=1,2, \ldots, \mathrm{z}^{*}$

\section{Appendix G}

Further results showing relative GFMAPR model performances on application to industrial and traffic accidents occurrence data (see Tables 23, 24, 25, 26, 27)

\section{References}

Aidoo SJ, Eshun PA (2012) Time series model of occupational injuries analysis in Ghanaian mines - a case study. Res J Environ Earth Sci 4(2):162-165

Arroyo J, Espínola R, Maté C (2011) Different approaches to forecast interval time series: a comparison in finance. Comput Econ 37(2):169-191
Arutchelvan G, Sivatsa SK, Jaganathan R (2010) Inaccuracy minimization by partitioning fuzzy data sets-validation of an analytical methodology. Int $J$ Comput Sci Inf Secur 8(1):275-280

Asrari A, Javan DS, Javidi MH, Monfared M (2012) Application of Gray-fuzzy-Markov chain method for day-ahead electric load forecasting. Przegląd Elektrotechniczny 88(3b):228-237

Brockwell PJ, Davis RA (2002) Introduction to time series and forecasting, 2nd edn. Springer, New York

Chen SM, Hsu CC (2004) A new method to forecast enrollments using fuzzy time series. Int J Appl Sci Eng 2(3):234-244

Cooke DL, Rohleder TR (2006) Learning from incidents: from normal accidents to high reliability. Sys Dyn Rev 22(3):213-239

Deng J (1982) Introduction to grey system theory. J Grey Syst 1:1-24

Docstoc (2013) Fire and explosions reported to BOEMRE. www. docstoc.com/docs/125138676/CY-2010-Fire-Explosions. 10 Aug 2016

Edem IE, Oke SA, Adebiyi KA (2016) A disjoint pair-point exponential and time-weighted-average fire accident forecasting model for partial information availability situations. Int J Manag Sci Eng Manag 11(3):155-166

Egrioglu E (2012) A new time-invariant fuzzy time series forecasting method based on genetic algorithm. Adv Fuzzy Syst. doi:10. $1155 / 2012 / 785709$

Farahat MA, Talaat M (2012) Short-term load forecasting using curve fitting prediction optimized by genetic algorithm. Int J Energy Eng 2(2):23-28

Garcia-Ascanio C, Maté C (2010) Electric power demand forecasting using interval time series: a comparison between VAR and iMLP. Energy Policy 38(2):715-725

Geng N, Zhang Y, Sun Y, Jiang Y, Chen D (2015) Forecasting China's annual biofuel production using an improved grey model. Energies 8:12087-12099

Hickey T, Ju Q, Van Emden MH (2001) Interval arithmetic: from principles to implementation. J ACM 48(5):1038-1068 
Huang P, Xuan X, Qiu R, Liu G (2012a) Statistical analysis on production safety accidents of heavy casualties of the period 2001-2011 in China. Procedia Eng 45:950-958

Huang GZ, Liu H, Yang CS, Wu ZG (2012b) Study on the model of construction accident forecast based on grey-Markov theory. Adv Mater Res 433-440:2874-2880

Jiang M-N (2007) Forecasting of fire accidents employing a reversed grey-Markov approach. Technol Forecast Soc Change 70(5):1272-1276

Jilani TA, Burney SMA (2008) Multivariate stochastic fuzzy forecasting models. Expert Syst Appl 35(3):691-700

Kamal SS, Gihan AE (2013) A new method for short multivariate fuzzy time series based on genetic algorithm and fuzzy clustering. doi:http://dx.doi.org/10.1155/2013/494239

Kang Y-S, Yang S-H, Kim T-G, Kim D-S (2012) Systematic strategies for the third industrial accident prevention plan in Korea. Ind Health 50:567-574

Kayacan E, Ulutas B, Kaynak O (2010) Grey systems theory based models in time series prediction. Expert Syst Appl 37:1784-1789

Kher AA, Yerpude RR (2015) Application of fuzzy time series forecasting model for indian underground coal mining fatal accident data. Int J Eng Technol 7(2):719-726

Kim T-G, Kang Y-S, Lee H-N (2011) A study on industrial accident rate forecasting and program development of estimated zero accident time in Korea. Ind Health 49:56-62

Lan J-Y, Ying Z (2014) Application of Gray Markov SCGM(1,1)c model to prediction of accidents deaths in coal mining. Int Sch Res Not. doi:http://dx.doi.org/10.1155/2014/632804

Lee LW, Wang LH, Chen SM (2007) Temperature prediction and TAIFEX forecasting based on fuzzy logical relationships and genetic algorithms. Expert Syst Appl 33(3):539-550

Li Y, Li M (2015) Prediction research of death number in construction accident based on unbiased grey-fuzzy-Markov chain method. In: 3rd Int conference on materials, mechanical and manuf eng (IC3ME 2015), pp 560-566

Liu S (2011) Interval analysis and grey systems theory. Springer, Berlin. link.springer.com/content/pdf/bbm\%3A978-3-64216158-2\%2F1.pdf. 13 Aug 2016

Liu S, Yang Y, Xie N, Forrest J (2016) New progress of grey system theory in the new millennium. Grey Sys Theory Appl 6(1):2-31

Mao M, Chirwa EC (2006) Application of grey model $\operatorname{GM}(1,1)$ to vehicle fatality risk estimation. Technol Forecast Soc Change 73:588-605

Mao ZL, Sun JH (2011) Application of grey-Markov model in forecasting fire accidents. Procedia Eng 11:314-318

Mohaghegh Z, Kazemi R, Mosceh A (2009) Incorporating organisational factors into probabilistic risk assessment (PRA) of complex socio-technical systems: a hybrid technique formalization. Reliab Eng Sys Saf 94:1000-1018
Mostafaei H, Kardooni S (2012) Hybrid grey forecasting for Iran's energy consumption supply. Int J Energy Econ 2(3):97-102

Ofori T, Ackah B, Ephraim L (2012) Statistical models for forecasting road accident injuries in Ghana. Int J Res Environ Sci Technol 2(4):143-149

Okoh P, Haugen S (2014) A study of maintenance related major accident cases in the 21st century. Process Saf Environ Prot 92:346-356

Oraee SK, Yazdani-Chamzini A, Basiri MH (2011) Forecasting the number of fatal injuries in underground coal mines. In: SME. 2011 SME annual meeting and exhibit and CMA 113th National Western Mining Conference "Shaping a Strong Future Through Mining", Denver, Colorado, USA

Qureshi ZH (2008) A review of accident modeling approaches for complex critical socio-technical systems. Technical Report DSTOTR-2094. In: Prepared for the command, control, communications and intelligence division, defence science and technology organisation (DSTO), Australia contact C31DDMO 07-007

Rathnayaka S, Khan F, Amyotte P (2011) SHIPP methodology: Predictive accident modelling approach. Part I: Methodology and model description. Process Saf Environ Prot 89:151-164

Shin IJ (2013) Major industrial accidents in Korea: the characteristics and implications of statistics 1996-2011. Process Saf Prog 32(1):91-95

Stellwagen E (2011) Forecasting 101: a guide to forecast error measurement statistics and how to use them. www.forecastpro. com/Trends/forecasting101August2011.html. 13 Aug 2016

Stringfellow MV (2010) Accident analysis and hazard analysis for human and organisational factors. Thesis submitted to the Department of Aeronautics and Astronautics, Massachusetts Institute of Technology, pp 1-283

Sullivan J, Woodall WH (1994) A comparison of fuzzy forecasting and Markov modelling. Fuzzy Sets Sys 64:279-293

Tangkuman S, Yang BJ (2011) Application of grey model for machine degradation prognostics. J Mech Sci Technol 25(12):2979-2985

Tong Q (2016) China's coal mine accident statistics analysis and one million tons mortality prediction. IETI Trans 2(1):61-72

Yabo X, Tong W, Bingxue S, Yushua X (2012) Forecasting of production safety situation by combination model. Procedia Eng 43:132-136

Zhang Y (2010) Predicting model of traffic volume based on greyMarkov. Mod Appl Sci 4(3):46-49

Zhao J, Ding J, Li F (2014) Major production accident interval forecasting based on gray panel. Procedia Eng 84:108-115

Zheng X, Liu M (2009) An overview of accident forecasting methodologies. J Loss Prev Process Ind 22:484-491 